\title{
Orthogonalities and functional equations
}

\author{
JUSTYNA SIKORSKA \\ Dedicated to Professor János Aczél on the occasion of his 90-th birthday
}

\begin{abstract}
In this survey we show how various notions of orthogonality appear in the theory of functional equations. After introducing some orthogonality relations, we give examples of functional equations postulated for orthogonal vectors only. We show their solutions as well as some applications. Then we discuss the problem of stability of some of them considering various aspects of the problem. In the sequel, we mention the orthogonality equation and the problem of preserving orthogonality. Last, but not least, in addition to presenting results, we state some open problems concerning these topics. Taking into account the big amount of results concerning functional equations postulated for orthogonal vectors which have appeared in the literature during the last decades, we restrict ourselves to the most classical equations.
\end{abstract}

Mathematics Subject Classification. 39B52, 39B82, 46B20, 47B49, 15A86.

Keywords. Orthogonality, Birkhoff orthogonality, James isosceles orthogonality, approximate orthogonality, inner product space, normed space, Hilbert modules, norm derivative, semiinner product, Cauchy equation, quadratic equation, exponential equation, orthogonal additivity, stability, orthogonality equation, orthogonality preserving property, linear preservers, isometry, approximate isometry.

\section{Contents}

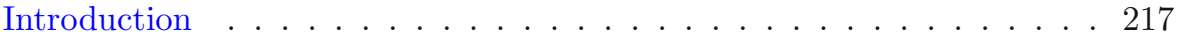

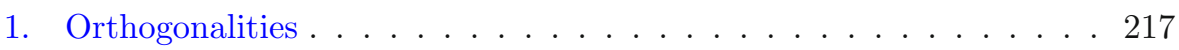

1.1. Various definitions of the orthogonality relation . . . . . . . . 217

1.1.1. Birkhoff orthogonality . . . . . . . . . . . . . . 217

1.1.2. Isosceles orthogonality . . . . . . . . . . . . . . 218

1.1.3. Pythagorean orthogonality . . . . . . . . . . . . 218

1.1.4. Norm derivatives orthogonality . . . . . . . . . . . . 218

1.1.5. Roberts orthogonality . . . . . . . . . . . . . 219

1.1.6. Semi-inner product orthogonality . . . . . . . . . . 219 
1.1.7. Diminnie orthogonality . . . . . . . . . . . . . . 219

1.1.8. Orthogonality space . . . . . . . . . . . . 220

1.1.9. Orthogonality defined via a difference operator . . . . 221

1.1.10. $C^{*}$-algebras . . . . . . . . . . . . 221

1.1.11. Orthogonalities on groups . . . . . . . . . . . . 221

1.2. Approximate orthogonalities . . . . . . . . . . . . . . 222

2. Functional equations postulated for orthogonal vectors . . . . . . 225

2.1. Cauchy functional equation . . . . . . . . . . . . . . 225

2.1.1. Solutions . . . . . . . . . . . . . . 225

2.1.2. Applications . . . . . . . . . . . . . . . . . 231

2.2. Jensen functional equation . . . . . . . . . . . . . . . 232

2.3. Quadratic functional equation . . . . . . . . . . . . . . 233

2.4. Exponential functional equation . . . . . . . . . . . 236

2.5. Other classical equations . . . . . . . . . . . . . . 238

2.5.1. D'Alembert equation . . . . . . . . . . . . . 238

2.5.2. Alternative Cauchy equation . . . . . . . . . . . . 239

2.5.3. Cocycle equation . . . . . . . . . . . . . . 240

2.6. Arithmetic functions . . . . . . . . . . . . . . . . . 240

3. Stability of functional equations postulated for orthogonal vectors . 241

3.1. Cauchy functional equation . . . . . . . . . . . . . . . 241

3.1.1. Hyers-Ulam stability . . . . . . . . . . . . . . . 241

3.1.2. Generalized stability . . . . . . . . . . . . . . 245

3.1.3. Some applications . . . . . . . . . . . . . . . . 246

3.1.4. Functional congruences . . . . . . . . . . . . . . 246

3.1.5. Orthogonal additivity almost everywhere . . . . . . . 250

3.1.6. Nearly additive set functions . . . . . . . . . . . . . 251

3.1.7. Arithmetic functions . . . . . . . . . . . . . . 251

3.2. Quadratic functional equation . . . . . . . . . . . . . 252

3.3. Exponential functional equation . . . . . . . . . . . . 254

3.3.1. Formulation of the stability problem . . . . . . . . 254

3.3.2. Multiplicative arithmetic functions . . . . . . . . 256

3.4. Other results . . . . . . . . . . . . . . . . . 257

4. Orthogonality equation . . . . . . . . . . . . 257

4.1. Stability of the orthogonality equation . . . . . . . 258

4.2. Orthogonality equation almost everywhere . . . . . . . . . 259

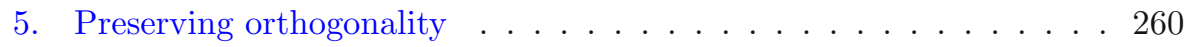

5.1. Exact orthogonality preserving mappings . . . . . . . . . 260

5.2. Mappings which approximately preserve orthogonality . . . 262

5.3. Stability of the orthogonality preserving property . . . . . 2 266

References . . . . . . . . . . . . . . . . . . . 269 


\section{Introduction}

During the last years many papers concerning various aspects of orthogonalities in the field of functional equations and inequalities have appeared. In this paper we want to give some overview on these results as well as to collect a number of items from the literature dealing with the subject. It is worth mentioning papers by Paganoni and Rätz [139] from 1995, Rätz [156] from 2001 and Chmieliński [42,44] from 2006, 2012, respectively, where the reader can find some partial collections of the results in this domain.

\section{Orthogonalities}

\subsection{Various definitions of the orthogonality relation}

As long as we are working in inner product spaces, usually there is no doubt what kind of orthogonality relation we have in mind. Namely, it is the one derived from an inner product and then vectors $x$ and $y$ are orthogonal $(x \perp y)$ if and only if $\langle x \mid y\rangle=0$. The situation looks completely different if we consider normed spaces or more general structures. We start the survey with listing some orthogonality relations described in normed spaces. More details and other orthogonality relations can be found in Amir [8], Alonso et al. [2,3], Alsina et al. [7], Alonso et al. [4], and in the references therein. Later on, we go on with some definitions in linear spaces, $C^{*}$-algebras and groups.

1.1.1. Birkhoff orthogonality. Let $(X,\|\cdot\|)$ be a real normed linear space. For vectors $x$ and $y$ from $X$, we say that $x$ is orthogonal to $y\left(x \perp_{B} y\right)$ in the sense of Birkhoff ( $x$ is Birkhoff orthogonal to $y$ ) if $\|x+\lambda y\| \geq\|x\|$ for all $\lambda \in \mathbb{R}$.

This orthogonality was introduced by Birkhoff [26], however since in normed linear spaces Birkhoff orthogonality is in fact equivalent to normality as it was introduced by Carathéodory, some ideas of this kind can already be found in Blaschke's book [28]. There are also other names for this orthogonality in the literature: Birkhoff-James orthogonality, Blaschke-Birkhoff-James orthogonality. James $[103,104]$ provided comprehensive studies on this relation. Many properties of this orthogonality relation are collected in Amir [8] and Alonso et al. [4].

If $X$ is an inner product space, then $\perp_{B}$ coincides with the standard orthogonality in the inner product space. Moreover, the Birkhoff orthogonality is homogeneous (i.e., if $x \perp_{B} y$, then $\alpha x \perp_{B} \beta y$ for all $\alpha, \beta$ in $\mathbb{R}$ ).

It is known (see Day [53], James [103]) that, if $\operatorname{dim} X \geq 3$, then $\perp_{B}$ is symmetric (i.e., $x \perp_{B} y$ if and only if $y \perp_{B} x$ ) if and only if $X$ is an inner product space. This result fails in two-dimensional spaces (see, e.g., Alonso et al. [4]). 
1.1.2. Isosceles orthogonality. James (see [102-104]) introduced in a normed linear space $(X,\|\cdot\|)$ the following orthogonality relation:

$$
x \perp_{J} y \quad \text { if and only if }\|x+y\|=\|x-y\| .
$$

The concept of this definition comes from the Euclidean plane, where a practical way to examine orthogonality between two vectors $x$ and $y$ is to check whether the two diagonals of the parallelogram determined by $x$ and $y$ are of equal length. The relation defined above is called James orthogonality or isosceles orthogonality.

When the norm $\|\cdot\|$ is derived from an inner product $\langle\cdot \mid \cdot\rangle$, then James orthogonality $x \perp_{J} y$ reduces to the classical condition $\langle x \mid y\rangle=0$. Moreover the James orthogonality $\perp_{J}$ is symmetric and partially homogeneous (i.e., if $x \perp_{J} y$, then $a x \perp_{J} a y$ for all $a$ in $\mathbb{R}$ ). Some other properties are collected in Amir [8] and Alonso et al. [4] and the references therein.

1.1.3. Pythagorean orthogonality. In a normed linear space $(X,\|\cdot\|)$ we say that $x$ is Pythagorean orthogonal to $y$ if and only if $\|x-y\|^{2}=\|x\|^{2}+\|y\|^{2}$. Similarly to the previously defined orthogonalities, this one was also studied in details by James [102].

At present, a variation of this orthogonality is more often used, namely the relation defined by

$$
x \perp_{P} y \quad \text { if and only if }\|x+y\|^{2}=\|x\|^{2}+\|y\|^{2}
$$

(see, e.g., Partington [141], Szabó [183], Alsina et al. [7]).

This orthogonality is symmetric, partially homogeneous, and in an inner product space it coincides with the orthogonality defined by means of the inner product.

Remark 1.1. Notwithstanding the way how the above orthogonalities were defined originally they may be considered also in complex normed spaces.

1.1.4. Norm derivatives orthogonality. Let $(X,\|\cdot\|)$ be a real normed linear space of dimension at least two. We consider the functions $\rho_{+}^{\prime}, \rho_{-}^{\prime}: X \times X \rightarrow \mathbb{R}$ defined as follows

$$
\rho_{ \pm}^{\prime}(x, y):=\lim _{t \rightarrow 0^{ \pm}} \frac{\|x+t y\|^{2}-\|x\|^{2}}{2 t} \text { for all } x, y \in X
$$

and call them norm derivatives. In an inner product space both functions $\rho_{+}^{\prime}$ and $\rho_{-}^{\prime}$ coincide with the inner product. Comprehensive studies of the properties of these functionals can be found in Alsina et al. [7] and Dragomir [60] (see also Amir [8]).

Let us now define the $\rho$-orthogonality relation $\perp_{\rho}$ by the condition

$$
x \perp_{\rho} y \quad \text { if and only if } \rho_{+}^{\prime}(x, y)+\rho_{-}^{\prime}(x, y)=0 .
$$

We will consider also $\rho_{+^{-}}$and $\rho_{-}$-orthogonality relations defined by

$$
x \perp_{\rho_{+}} y \quad \text { if and only if } \rho_{+}^{\prime}(x, y)=0
$$


and

$$
x \perp_{\rho_{-}} y \text { if and only if } \rho_{-}^{\prime}(x, y)=0,
$$

respectively. Among the just defined three orthogonality relations only $\perp_{\rho}$ is homogeneous, and none of them is symmetric.

For the further considerations, let us denote $\rho^{\prime}:=\frac{1}{2}\left(\rho_{+}^{\prime}+\rho_{-}^{\prime}\right)$ (see Miličić [129], Dragomir [60]).

1.1.5. Roberts orthogonality. It seems that Roberts was the first who introduced the orthogonality relation in normed linear spaces. Namely, he proposed his definition of orthogonality in 1934 (see [161]). We say that $x$ is orthogonal to $y$ in the sense of Roberts $\left(x \perp_{R} y\right)$ if and only if $\|x+t y\|=\|x-t y\|$ for all $t \in \mathbb{R}$.

It is obvious that this orthogonality implies both James and Birkhoff orthogonalities. Moreover, Roberts orthogonality is symmetric.

1.1.6. Semi-inner product orthogonality. Let $(X,\|\cdot\|)$ be a normed linear space. A functional $[\cdot \mid \cdot]: X \times X \rightarrow \mathbb{K} \in\{\mathbb{R}, \mathbb{C}\}$ satisfying

- $[\lambda x+\mu y \mid z]=\lambda[x \mid z]+\mu[y \mid z]$ for all $x, y, z \in X$ and $\lambda, \mu \in \mathbb{K}$;

- $[x \mid \lambda y]=\bar{\lambda}[x, y]$ for all $x, y \in X$ and $\lambda \in \mathbb{K}$;

- $[x, x]=\|x\|^{2}$ for all $x \in X$;

- $|[x, y]| \leq\|x\|\|y\|$ for all $x, y \in X$

is called a semi-inner product in a normed space $X$ (generating the given norm). Lumer [124] and Giles [91] proved that in any normed space there exists a semi-inner product. There can be infinitely many such semi-inner products. It is known, however, that in a normed space there exists exactly one semi-inner product if and only if the space is smooth (which means that the norm in $X$ is smooth, that is, it is Gâteaux differentiable)(see, e.g., Day [54]).

For a given semi-inner product and vectors $x, y \in X$ we define the semiinner product orthogonality

$$
x \perp_{s} y \quad \text { if and only if } \quad[y \mid x]=0 .
$$

1.1.7. Diminnie orthogonality. Let $(X,\|\cdot\|)$ be a real normed linear space, and let $F:=\left\{\varphi \in X^{*}:\|\varphi\| \leq 1\right\}$. Define

$$
\|x, y\|=\sup \left\{\left|\begin{array}{ll}
\varphi(x) & \varphi(y) \\
\psi(x) & \psi(y)
\end{array}\right|: \varphi, \psi \in F\right\} \quad \text { for all } x, y \in X .
$$

In 1983, Diminnie [56] proposed the following orthogonality relation

$$
x \perp_{D} y \quad \text { if and only if } \quad\|x, y\|=\|x\| \cdot\|y\| .
$$


He described its connections to Birkhoff orthogonality and proved that if $\operatorname{dim} X \geq 3$ then merely the additivity of the relation or the fact that the inequality $\|x, y\| \leq\|x\| \cdot\|y\|$ holds true for all $x, y \in X$ characterize $X$ as an inner product space.

1.1.8. Orthogonality space. Apart from the different definitions of orthogonalities in normed spaces we may give some axiomatic definition of such a relation in linear spaces. The most often cited definition of an orthogonality space is the one given by Rätz [149]:

Definition 1.1. Let $X$ be a real linear space with $\operatorname{dim} X \geq 2$ and let $\perp$ be a binary relation on $X$ such that

(01) $x \perp 0$ and $0 \perp x$ for all $x \in X$;

(02) if $x, y \in X \backslash\{0\}$ and $x \perp y$, then $x$ and $y$ are linearly independent;

(03) if $x, y \in X$ and $x \perp y$, then for all $\alpha, \beta \in \mathbb{R}$ we have $\alpha x \perp \beta y$;

(04) for any two-dimensional subspace $P$ of $X$ and for every $x \in P, \lambda \in[0, \infty)$, there exists $y \in P$ such that $x \perp y$ and $x+y \perp \lambda x-y$.

An ordered pair $(X, \perp)$ is called an orthogonality space in the sense of Rätz, or shortly, orthogonality space.

This definition is more restrictive than the ones given before by Gudder and Strawther (see $[94,95]$ ), however, none of the examples provided by them is omitted while considering the definition by Rätz. In [94], the authors define $\perp$ by (01)-(03) and add

$\left(04^{\prime}\right)$ for every two-dimensional subspace $P$ of $X$ and for every nonzero $x \in P$, there exists a nonzero $y \in P$ such that $x \perp y$ and $x+y \perp x-y$.

In [95], together with (01)-(03) there are

$\left(04^{\prime \prime}\right)$ if $P$ is a two-dimensional subspace of $X$, then for every $x \in P$, there exists a nonzero $y \in P$ such that $x \perp y$;

(05) if $P$ is a two-dimensional subspace of $X$, then there exist nonzero vectors $x, y \in P$ such that $x \perp y$ and $x+y \perp x-y$.

An orthogonality space covers the case of an inner product space with the classical orthogonality as well as an arbitrary real normed linear space with the Birkhoff orthogonality. But it is also the case with the "trivial" orthogonality defined on a linear space by (01) and the condition that two nonzero vectors are orthogonal if and only if they are linearly independent.

However, there are known orthogonality relations on normed linear spaces which do not satisfy axioms (01)-(04), e.g., the isosceles orthogonality and the Pythagorean orthogonality.

In the following papers Rätz [150,151] and then Rätz and Szabó [157] developed the theory by considering various generalizations of the stated definitions (see also Szabó [179]). 
1.1.9. Orthogonality defined via a difference operator. Given a real functional $\varphi$ on an Abelian group $(X,+)$ we may define a new orthogonality relation by the formula

$$
x \perp_{\varphi} y \text { if and only if } \triangle_{x, y} \varphi(z)=0 \text { for all } z \in X,
$$

where $\triangle_{x}$ denotes the usual difference operator, $\triangle_{x} \varphi(z)=\varphi(x+z)-\varphi(z)$, and $\triangle_{x, y}=\triangle_{x} \circ \triangle_{y}$ for all $x, y \in X$.

The above orthogonality relation was proposed by Ger in [87]. It generalizes the trapezoid orthogonality $\perp_{T}$ on a normed space defined by Alsina et al. in [5] by

$$
\begin{aligned}
& x \perp_{T} y \text { if and only if }\|z-x\|^{2}+\|z-y\|^{2}=\|z\|^{2}+\|z-(x+y)\|^{2} \\
& \quad \text { for all } z \in X .
\end{aligned}
$$

It means that if $X$ is a real normed space and $\varphi=\|\cdot\|^{2}, \varphi$-orthogonality coincides with the T-orthogonality.

Ger [87] studies the properties of the relation $\perp_{\varphi}$ and obtains some new characterizations of inner product spaces, e.g., if $X$ is a linear topological space and $\varphi$ is a continuous functional, $\left(X, \perp_{\varphi}\right)$ is an orthogonality space if and only if $X$ is an inner product space (i.e., there exists an inner product $\langle\cdot \mid \cdot\rangle: X^{2} \rightarrow \mathbb{R}$ such that $\|x\|=\sqrt{\langle x \mid x\rangle}$ for all $x \in X)$.

1.1.10. $C^{*}$-algebras. Suppose $\mathcal{A}$ is a $C^{*}$-algebra. Let $X$ be an algebraic right $\mathcal{A}$-module which is a complex linear space with a compatible scalar multiplication, i.e., $(\lambda x) a=x(\lambda a)=\lambda(x a)$ for all $x \in X, a \in \mathcal{A}, \lambda \in \mathbb{C}$.

Then $X$ is called a (right) inner product $\mathcal{A}$-module if there exists an $\mathcal{A}$ valued inner product, i.e., a mapping $\langle\cdot \mid \cdot\rangle: X \times X \rightarrow \mathcal{A}$ satisfying

- $\langle x \mid x\rangle \geq 0$ (positive element of $\mathcal{A}$ ) and $\langle x \mid x\rangle=0$ if and only if $x=0$;

- $\langle x \mid \lambda y+z\rangle=\lambda\langle x \mid y\rangle+\langle x \mid z\rangle$

- $\langle x \mid y a\rangle=\langle x \mid y\rangle a$

- $\langle y \mid x\rangle=\langle x \mid y\rangle^{*}$

for all $x, y, z \in X, a \in \mathcal{A}, \lambda \in \mathbb{C}$ (cf., e.g., Lance [121]).

The orthogonality relation in $X$ is naturally defined by

$$
x \perp y \quad \text { if and only if }\langle x \mid y\rangle=0 .
$$

1.1.11. Orthogonalities on groups. In 1998, Baron and Volkmann [24] proposed the following axioms of orthogonality. Let $(X,+)$ be a uniquely 2 divisible Abelian group. Further, let $\perp$ be a binary relation defined on $X$ with the properties:

(a) $0 \perp 0$;

(b) if $x, y \in X$ and $x \perp y$, then $-x \perp-y$ and $\frac{x}{2} \perp \frac{y}{2}$; 
(c) every odd orthogonally additive mapping having values in an Abelian group is additive and every even orthogonally additive mapping is quadratic (see Sect. 2.1.1).

In 2010, Fechner and Sikorska [69] were dealing with the stability of orthogonal additivity (see Sect. 3.1.1) proposing the following definition of orthogonality: Let $(X,+)$ be an Abelian group and let $\perp$ be a binary relation defined on $X$ with the properties:

( $\alpha$ ) if $x, y \in X$ and $x \perp y$, then $x \perp-y,-x \perp y$ and $2 x \perp 2 y$;

$(\beta)$ for every $x \in X$, there exists $y \in X$ such that $x \perp y$ and $x+y \perp x-y$.

Each orthogonality space satisfies these conditions as well as an arbitrary normed linear space with the isosceles orthogonality, but it is no longer the case with the Pythagorean orthogonality.

In what follows, we provide an example of a binary relation which seems to be far from any known orthogonality relations but satisfies $(\alpha)$ and $(\beta)$.

Example 1.1. (Fechner and Sikorska [69]) Take $X=\mathbb{R}$ and define $\perp \subset \mathbb{R}^{2}$ in the following way:

$$
x \perp y \quad \text { if and only if } \quad x \cdot y \in \mathbb{R} \backslash \mathbb{Q} \text { or } x \cdot y=0 .
$$

Considering usually at least two-dimensional spaces while dealing with orthogonalities allows us to avoid trivial situations, i.e., situations when $x \perp y$ implies that $x=0$ or $y=0$. In the above example it is not the case. However, of course this example can be extended to higher dimensional inner product spaces, where the set of "orthogonal" vectors is considerably bigger than in the standard case.

\subsection{Approximate orthogonalities}

Let $\varepsilon \in[0,1)$. A natural way to define approximate orthogonality (or $\varepsilon$ orthogonality) of vectors $x$ and $y$ in an inner product space is:

$$
x \perp^{\varepsilon} y \quad \text { if and only if }|\langle x \mid y\rangle| \leq \varepsilon\|x\|\|y\| .
$$

Quite similarly, in normed spaces we define the approximate semi orthogonality relation ( $\varepsilon$-semi-inner product orthogonality) and approximate $\rho$-orthogonality ( $\varepsilon$ - $\rho$-orthogonality) as follows

$$
x \perp_{s}^{\varepsilon} y \quad \text { if and only if } \quad|[y \mid x]| \leq \varepsilon\|x\|\|y\|
$$

and

$$
x \perp_{\rho}^{\varepsilon} y \quad \text { if and only if } \quad\left|\rho^{\prime}(x, y)\right| \leq \varepsilon\|x\|\|y\|,
$$

respectively (see Dragomir [59], and also Chmieliński [43], Chmieliński and Wójcik [49]). Analogous definitions to (1.1) are obtained with $\rho$ replaced by $\rho_{+}$or $\rho_{-}$. 
There are two notions of approximate Birkhoff orthogonality (motivations for using such relations are described in Mojškerc and Turnšek [134]). The first one comes from Dragomir [58,59]:

$$
x^{\varepsilon} \perp_{B} y \quad \text { if and only if }\|x+\lambda y\| \geq(1-\varepsilon)\|x\| \text { for all } \lambda \in \mathbb{K} .
$$

For inner product spaces we have (Dragomir [59]; see also Chmieliński [44])

$$
x^{\varepsilon} \perp_{B} y \text { if and only if } x \perp^{\delta} y \text {, where } \delta=\sqrt{(2-\varepsilon) \varepsilon} .
$$

Another definition of approximate Birkhoff orthogonality (generally not equivalent to the just mentioned one) comes from Chmieliński [40]:

$$
x \perp_{B}^{\varepsilon} y \text { if and only if }\|x+\lambda y\|^{2} \geq\|x\|^{2}-2 \varepsilon\|x\|\|\lambda y\| \text { for all } \lambda \in \mathbb{K} .
$$

Mojškerc and Turnšek [134] showed that for any $x, y$ from a normed space (real or complex) the relation $x \perp_{B}^{\varepsilon} y$ implies $x{ }^{\delta} \perp_{B} y$, where $\delta=1-\sqrt{1-4 \varepsilon}$, and the converse holds (with some $\varepsilon$ depending on $\delta$ ), e.g., in uniformly smooth spaces.

Moreover, we have the following properties.

Theorem 1.1. (Chmieliński and Wójcik $[49,50]$ ) For an arbitrary real normed linear space $X$ and $\varepsilon \in[0,1)$ we have

$$
\perp_{\rho_{+}}^{\varepsilon} \subset \perp_{B}^{\varepsilon}, \quad \perp_{\rho_{-}}^{\varepsilon} \subset \perp_{B}^{\varepsilon}, \quad \perp_{\rho}^{\varepsilon} \subset \perp_{B}^{\varepsilon}, \quad \perp_{s}^{\varepsilon} \subset \perp_{B}^{\varepsilon}
$$

(with the standard notation, that is, e.g., $\perp_{B}^{\varepsilon}$ denotes the set $\left\{(x, y) \in X^{2}\right.$ : $\left.\left.x \perp_{B}^{\varepsilon} y\right\}\right)$. Moreover, if the norm is smooth, then $\perp_{\rho}^{\varepsilon}=\perp_{B}^{\varepsilon}=\perp_{s}^{\varepsilon}$.

Theorem 1.2. (Chmieliński and Wójcik [50]) Let $(X,\|\cdot\|)$ be an arbitrary real normed linear space and let $\varepsilon \in[0,1)$. Then for arbitrary $x, y \in X$ and $\alpha \in \mathbb{R}$ we have

$$
\begin{aligned}
& x \perp_{B}^{\varepsilon}(y-\alpha x) \quad \Leftrightarrow \quad \rho_{-}^{\prime}(x, y)-\varepsilon\|x\|\|y-\alpha x\| \\
& \leq \alpha\|x\|^{2} \leq \rho_{+}^{\prime}(x, y)+\varepsilon\|x\|\|y-\alpha x\| .
\end{aligned}
$$

As a special case of the latter result we obtain the property:

$$
x \perp_{B}^{\varepsilon} y \quad \Leftrightarrow \quad \rho_{-}^{\prime}(x, y)-\varepsilon\|x\|\|y\| \leq 0 \leq \rho_{+}^{\prime}(x, y)+\varepsilon\|x\|\|y\| .
$$

So, we have generalizations of the known conditions:

$$
x \perp_{B}(y-\alpha x) \quad \Leftrightarrow \quad \rho_{-}^{\prime}(x, y) \leq \alpha\|x\|^{2} \leq \rho_{+}^{\prime}(x, y)
$$

and

$$
x \perp_{B} y \quad \Leftrightarrow \quad \rho_{-}^{\prime}(x, y) \leq 0 \leq \rho_{+}^{\prime}(x, y)
$$

(see, e.g., Amir [8]).

Two notions of approximate James orthogonality are given by the following conditions (see Chmieliński and Wójcik [48]): 
$x \perp_{J}^{\varepsilon} y \quad$ if and only if $\quad\left|\|x+y\|^{2}-\|x-y\|^{2}\right| \leq 4 \varepsilon\|x\|\|y\|$,

and

$x^{\varepsilon} \perp_{J} y \quad$ if and only if $\quad\|x+y\|-\|x-y\| \mid \leq \varepsilon(\|x+y\|+\|x-y\|)$, or equivalently,

$$
x^{\varepsilon} \perp_{J} y \quad \text { if and only if } \quad \frac{1-\varepsilon}{1+\varepsilon}\|x-y\| \leq\|x+y\| \leq \frac{1+\varepsilon}{1-\varepsilon}\|x-y\| .
$$

Obviously, for $\varepsilon=0$ both versions of the approximate J-orthogonality coincide with the J-orthogonality. As observed by Chmieliński and Wójcik in [48], the second definition of approximate J-orthogonality is weaker than the first one, i.e., for an arbitrary $\varepsilon \in[0,1)$ the condition $x \perp_{J}^{\varepsilon} y$ implies $x{ }^{\varepsilon} \perp_{J} y$, but not conversely.

One can check that in the case when the norm comes from a real valued inner product, then

$$
x \perp_{J}^{\varepsilon} y \Leftrightarrow|\langle x \mid y\rangle| \leq \varepsilon\|x\|\|y\| \Leftrightarrow x \perp^{\varepsilon} y
$$

and

$$
x^{\varepsilon_{J}} y \Leftrightarrow|\langle x \mid y\rangle| \leq \frac{\varepsilon}{1+\varepsilon^{2}}\left(\|x\|^{2}+\|y\|^{2}\right)
$$

So, the first (stronger) approximate J-orthogonality coincides with the standard notion of approximate orthogonality in inner product spaces.

Example 1.2. Let $(X,\langle\cdot \mid \cdot\rangle)$ be an inner product space, $x \in X \backslash\{0\}$ and $y=\lambda x$ for some $\lambda>0$. Then $\frac{|\langle x \mid y\rangle|}{\|x\|^{2}+\|y\|^{2}}=\frac{\lambda}{1+\lambda^{2}} \rightarrow 0$ as $\lambda \rightarrow \infty$, and $\frac{|\langle x \mid y\rangle|}{\|x\|\|y\|}=1$ for all $\lambda$. Thus for arbitrary $x \neq 0$ and $\varepsilon \in[0,1)$ there exists $\lambda$ such that $x^{\varepsilon} \perp_{J} \lambda x$ whereas $x \perp_{J}^{\varepsilon} \lambda x$ does not hold for any $\varepsilon \in[0,1)$.

It is known that the conditions $\perp_{B} \subset \perp_{J}$ or $\perp_{J} \subset \perp_{B}$ characterize $(X,\|\cdot\|)$ as an inner product space, so it is quite natural to ask about the connections between approximate James orthogonalities and approximate Birkhoff orthogonalities.

Finally, similarly to Chmieliński and Wójcik [48], two notions of approximate Roberts orthogonality are given by Zamani and Moslehian [201]:

$x \perp_{R}^{\varepsilon} y \quad$ if and only if $\quad\left|\|x+t y\|^{2}-\|x-t y\|^{2}\right| \leq 4 \varepsilon\|x\|\|t y\|$ for all $t \in \mathbb{R}$, and

$x^{\varepsilon} \perp_{R} y \quad$ if and only if $\quad|\|x+t y\|-\|x-t y\|| \leq \varepsilon(\|x+t y\|+\|x-t y\|)$ for all $t \in \mathbb{R}$. 


\section{Functional equations postulated for orthogonal vectors}

\subsection{Cauchy functional equation}

2.1.1. Solutions. We start this section with giving some historical background for the investigations in this field (see also Paganoni and Rätz [139], Rätz [156]).

In what follows we will consider the Cauchy functional equation postulated for orthogonal vectors only, that is the conditional equation

$$
f(x+y)=f(x)+f(y) \text { for all } x, y \text { with } x \perp y
$$

(the domain, target space and the orthogonality relation will be specified later). Functions satisfying (2.1) are called orthogonally additive.

The studies on (2.1) were begun (to the best of our knowledge) by Pinsker in 1938 who was considering (see [143]) orthogonally additive mappings defined on the space of continuous functionals from $L^{2}[a, b]$ with an orthogonality defined by

$$
x \perp y \quad \text { if and only if } \quad \int_{a}^{b} x(t) y(t) d t=0
$$

(so by means of the inner product). Later on, the studies proceeded in two directions concerning the domain. It was considered: (i) a set of continuous functions on some type of topological space or (ii) a set of measurable functions on a measure space. Then we say that $x, y$ are orthogonal in the lattice theoretic sense $\left(x \perp_{L} y\right)$ if the set $\{t: x(t) y(t)=0\}$ is empty in case (i) or of measure zero in case (ii). A real valued functional $f$ is L-additive if $f(x+y)=f(x)+f(y)$ whenever $x \perp_{L} y$. If $f$ is $L$-additive and satisfies certain continuity or boundedness conditions, then $f$ admits an integral representation giving a nonlinear generalization of the Riesz theorem. Such representations have been obtained for case (i) in [36,79] and for case (ii) in [61,78,126,131,132,174].

The above mentioned concepts of orthogonality are quite natural in the spaces considered and they are important for certain applications [81,123, 159], however, there are several other concepts of orthogonality defined and developed by Birkhoff, Roberts, James and Day (see Sect. 1).

We restrict our attention to the approach (being in fact the continuation of Pinsker's [143]) which was begun in 1972 by Sundaresan [175]. He studied orthogonally additive functions defined on an inner product space or on a normed linear space with Birkhoff orthogonality, and he gave some partial results in the case of continuous orthogonally additive functions. His main result reads as follows.

Theorem 2.1. (Sundaresan [175]) Let $f$ be a continuous function from a Banach space $(X,\|\cdot\|)$ into a locally convex space $Y$. If $X$ is a Hilbert space, then $f$ is 
orthogonally additive if and only if there exist a vector $c \in Y$ and a continuous linear operator $h: X \rightarrow Y$ such that

$$
f(x)=\|x\|^{2} \cdot c+h(x) \quad \text { for all } x \in X .
$$

Moreover, if $X$ is not isometric with a Hilbert space (that is, there is no bilinear symmetric inner product in it from which the given norm can be derived in the customary way), then $f$ is a continuous linear operator on $X$ into $Y$.

Studies were continued by Gudder and Strawther [94-96] and Dhombres [55]. The manuscript [94] from 1974 contains a collection of properties and results without proofs, but we can find there the first axiomatization of the orthogonality relation containing the former situations as special cases. In the next paper [95], bringing a slightly different axiomatic definition, the authors already gave some explanations and showed the form of real solutions of (2.1) under some boundedness conditions. For the main results the assumption about the completeness of the domain turned out to be superfluous.

Theorem 2.2. (Gudder and Strawther [95]) (Characterization of inner product spaces (i.p.s.)) Let $(X, \perp)$ be an orthogonality space (defined by (01)-(03) and (04')). If there exists $f: X \rightarrow \mathbb{R}$ which is even, orthogonally additive, hemicontinuous ${ }^{1}$ and not identically zero, then there is an inner product $\langle\cdot \mid \cdot\rangle$ on $X$ such that for any $x, y \in X, x \perp y$ if and only if $\langle x \mid y\rangle=0$. In fact, $\langle x \mid y\rangle=\frac{1}{4}[f(x+y)-f(x-y)]$ and the induced norm satisfies $\|x\|^{2}=f(x)$ for all $x, y \in X$ or $\|x\|^{2}=-f(x)$ for all $x \in X$.

As a corollary, Gudder and Strawther [95] obtained an analogous form of solutions as in Theorem 2.1 for real functions defined on a normed space with the Birkhoff orthogonality under the assumption of hemicontinuity. Moreover, they proved a generalization of the Riesz representation theorem, showing that if $X$ is an inner product space and $f: X \rightarrow \mathbb{R}$ is orthogonally additive and satisfies $|f(x)| \leq M\|x\|$ for all $x \in X$, then $f$ is a continuous linear functional and hence, if $X$ is a Hilbert space, then $f(x)=\langle x \mid z\rangle$ for some $z \in X$.

In his book [55], Dhombres states the open problem whether the regularity assumption of a considered function may be omitted in order to derive that in a normed space of dimension not less than 2 the existence of an even nonzero orthogonally additive mapping characterizes inner product spaces.

So, the next step was to get rid of the regularity conditions and to characterize in an abstract framework - the general even and the general odd solution of (2.1) with values in an Abelian group (Lawrence [122], Rätz [149-151], Szabó [176], Rätz and Szabó [157]). Roughly speaking, in many important situations, the general even solution is quadratic, and the general odd solution is additive.

\footnotetext{
${ }^{1}$ A function $f: X \rightarrow \mathbb{R}$ is called hemicontinuous at $x_{0} \in X$ if and only if the function $\mathbb{R} \ni \alpha \mapsto f\left(\alpha x_{0}\right)$ is continuous, and it is called hemicontinuous if it is hemicontinuous at every $x \in X$.
} 
Theorem 2.3. (Rätz [149]) Let $(X, \perp)$ be an orthogonality space and $(Y,+)$ be a uniquely 2-divisible Abelian group. If $f: X \rightarrow Y$ is a solution of (2.1), then it has the form $f=a+q$, where $a$ is additive and $q$ is quadratic.

Theorem 2.4. (Rätz [147-149]) Let $(X,\|\cdot\|)$ be an inner product space and $(Y,+)$ be a uniquely 2-divisible Abelian group. Then $f: X \rightarrow Y$ is a solution of (2.1) if and only if there exist additive mappings $a: X \rightarrow Y$ and $b: \mathbb{R} \rightarrow Y$ such that

$$
f(x)=a(x)+b\left(\|x\|^{2}\right) \text { for all } x \in X .
$$

The above result in the case of a uniquely 2-divisible Abelian group $(Y,+)$ was found independently by Ger and Szabó (see [84]). Under the assumption that $(Y,+)$ is 2-torsion-free it was proved by Rätz and Szabó in [157].

Baron and Rätz in [23] (for inner product spaces) and then Baron and Volkmann in [24] showed that the assumption that $Y$ is uniquely 2-divisible as well as the 2-torsion-freeness of $Y$ may be omitted. We present here a theorem from [24].

Theorem 2.5. (Baron and Volkmann [24]) Let $X$ be a linear space over a field of characteristic different from 2 (or let $X$ be a uniquely 2-divisible group), $(Y,+)$ be an Abelian group and let $f: X \rightarrow Y$. Assume that $\perp$ is a binary relation on $X$ such that

(i) $0 \perp 0$ and $x, y \in X, x \perp y$, implies $-x \perp-y$ and $\frac{x}{2} \perp \frac{y}{2}$;

(ii) every odd orthogonally additive mapping from $X$ to $Y$ is additive and every even orthogonally additive mapping is quadratic.

Then $f$ is orthogonally additive if and only if

$$
f(x)=a(x)+b(x, x) \text { for all } x \in X,
$$

with $a: X \rightarrow Y$ being additive and $b: X \times X \rightarrow Y$ being biadditive, symmetric and such that $b(x, y)=0$ whenever $x \perp y$.

Moreover, in this case the functions $a, b$ and $q(x)=b(x, x), x \in X$, are uniquely determined; they are given by

$$
\begin{aligned}
& a(x)= f\left(\frac{x}{2}\right)-f\left(-\frac{x}{2}\right) \quad \text { for all } x \in X, \\
& b(x, y)=2\left[f\left(\frac{x+y}{4}\right)+f\left(\frac{-x-y}{4}\right)-f\left(\frac{x-y}{4}\right)-f\left(\frac{-x+y}{4}\right)\right] \quad \text { for all } x, y \in X, \\
& q(x)=2\left[f\left(\frac{x}{2}\right)+f\left(-\frac{x}{2}\right)\right] \quad \text { for all } x \in X,
\end{aligned}
$$

respectively.

If $X$ is an inner product space then various assumptions force orthogonally additive functions $f$ to be of the form (2.2), namely: 
- $\quad Y$ is a separated topological $\mathbb{R}$-vector space and $f$ is continuous (Rätz [149, Corollary 11]; cf. Theorem 2.1 and Sundaresan [175]);

- $\quad f: X \rightarrow \mathbb{R}$ satisfies $|f(x)| \leq m\|x\|$ for all $x \in X$ and a fixed $m \geq 0$ (Gudder and Strawther [95, Corollary 2.4], Rätz [149, Corollary 12]);

- $\quad f: X \rightarrow \mathbb{R}$ satisfies $f(x) \geq 0$ for all $x \in X$; then we obtain (2.2) with $h=0$ and with nonnegative $c$ (see Gudder and Strawther [96]; Rätz [149, Corollary 13]);

- $\quad f: X \rightarrow \mathbb{R}$ is bounded on a second category Baire subset of $X$ (follows from Rätz [149], Ger [82]; see also Ger and Sikorska [89]);

- $Y$ is a topological Abelian group and $f: X \rightarrow Y$ is continuous at a point (Baron and Kucia [21, Theorem 4.3]).

The last result was generalized first by Brzdęk [32] (with the domain being an orthogonality space and with the assumption of continuity at the origin) and then by Wyrobek [198] who was working in an Abelian topological group in the domain with the assumption of continuity at an arbitrary point.

It is possible to characterize Hilbert spaces among real inner product spaces in terms of the boundedness behavior of $\mathbb{R}$-valued orthogonally additive mappings. The result is related to the Riesz representation theorem.

Theorem 2.6. (Rätz [149]) (Characterization of Hilbert spaces) For an inner product space $X$ the following conditions are equivalent:

(i) for every orthogonally additive mapping $f: X \rightarrow \mathbb{R}$ which is bounded from below, there exists an element $x_{0} \in X$ such that $f\left(x_{0}\right) \leq f(x)$ for all $x \in X$;

(ii) $X$ is a Hilbert space.

For several years mathematicians were trying to find the connection between the property $(\mathrm{e}) \mathrm{Hom}_{\perp}(X, Y)=\{0\}$ (where $(\mathrm{e}) \mathrm{Hom}_{\perp}(X, Y)$ stands for the set of all even orthogonally additive functions from $X$ to $Y$ ) and the property that $X$ is an inner product space.

Theorem 2.7. (Rätz [149], Szabó [176]) (Characterization of i.p.s.) Let (X,\|·\|) be a real normed space, $\operatorname{dim} X \geq 2$, with Birkhoff orthogonality $\perp_{B}$ and $(Y,+)$ be an Abelian group. $(X,\|\cdot\|)$ is an inner product space if and only if not every $\perp_{B}$-additive mapping $f: X \rightarrow Y$ is additive.

In [149], the above theorem is proved for at least three-dimensional spaces. Moreover, in [176], it is additionally proved that if $Y$ is an arbitrary group (so not necessarily Abelian), then every even orthogonally additive mapping is identically zero.

The following fact concerns the symmetry of relation $\perp$. It is known that in at least three dimensional normed spaces $X$, the symmetry of $\perp_{B}$ characterizes $X$ as an inner product space. Lawrence [122] proved that if $\operatorname{dim} X=2$ and $\perp_{B}$ is not symmetric, then every orthogonally additive mapping is additive. In fact, we can prove this result in an arbitrary orthogonality space. 
Theorem 2.8. (Rätz [150]) For any orthogonality space $(X, \perp)$ and any Abelian group $(Y,+)$, if $(\mathrm{e}) \operatorname{Hom}_{\perp}(X, Y) \neq\{0\}$, then $\perp$ is symmetric.

In 1990, Szabó proved the following (see [177]).

Theorem 2.9. Let $(X, \perp)$ be an orthogonality space and let $(Y,+)$ be an Abelian group. If $\operatorname{dim} X \geq 3$ and there is a nontrivial even orthogonally additive mapping $f: X \rightarrow Y$, then $X$ is an inner product space.

In [179], Szabó went on trying to answer the question about dimension 2. By strengthening the fourth assumption in the definition of orthogonality space he proved that the above result is also true when $\operatorname{dim} X=2$. In 2001 , Rätz [156] came back to the problem and asked whether it is also true in arbitrary orthogonality spaces $X$ with $\operatorname{dim} X=2$.

An affirmative answer was given by Yang in 2006. She showed (see [200])

Theorem 2.10. (Characterization of i.p.s.) Suppose $(X, \perp)$ is an orthogonality space and there exists a nontrivial even orthogonally additive function $f: X \rightarrow$ $Y$ for some Abelian group $(Y,+)$. Then $X$ is an inner product space.

Interesting results were obtained lately by Baron [16-18]. He was working with orthogonally additive involutions, functions with orthogonally additive second iterate, finally with orthogonally additive bijections in real inner product spaces.

Theorem 2.11. Any orthogonally additive bijection from a real inner product space into an Abelian group is additive.

In [190], Turnšek extended this result to complex or quaternionic inner product spaces.

The following examples show that none of the assumptions: injectivity or surjectivity, may be omitted (see Baron [16]).

Example 2.1. Assume that $X$ is a real inner product space. Let $H_{0}$ be a basis of $\mathbb{R}$ over $\mathbb{Q}$ and let $H$ be a basis of $X$ over $\mathbb{Q}$. If $H_{1}$ and $H_{2}$ are disjoint subsets of $H$ such that

$$
1 \leq \operatorname{card} H_{1} \leq \mathfrak{c} \text { and } \operatorname{card} H_{2}=\operatorname{card} H,
$$

and $a: \mathbb{R} \rightarrow X$ and $b: X \rightarrow X$ are additive functions such that $a\left(H_{0}\right)=H_{1}$, $b(H)=H_{2}$ and $b$ is injective, then $f: X \rightarrow X$ given by (2.3) is orthogonally additive, injective and it is not additive.

Example 2.2. Assume $X=X_{1} \oplus X_{2}, X_{1} \perp X_{2}$ and $\operatorname{dim} X_{1}=1$. Fix $e \in X_{1}$ with $\|e\|=1$, let $a_{0}: \mathbb{R} \rightarrow \mathbb{R}$ and $b_{0}: X_{2} \rightarrow X_{2}$ be additive functions such that $a_{0}([0, \infty))=\mathbb{R}, b_{0}\left(X_{2}\right)=X_{2}$ and define $a: \mathbb{R} \rightarrow X$ and $b: X \rightarrow X$ by

$$
a(\alpha)=a_{0}(\alpha) e, \quad b\left(\alpha e+x_{2}\right)=b_{0}\left(x_{2}\right) \quad \text { for all } \alpha \in \mathbb{R} \text { and } x_{2} \in X_{2} .
$$

Then the function $f: X \rightarrow X$ given by (2.3) is orthogonally additive, $f(X)=$ $X$ and $f$ is not additive. 
Theorem 2.12. (Baron [17]) Suppose $(X,\langle\cdot \mid \cdot\rangle)$ is a real inner product space. Assume $f: X \rightarrow X$ and $f^{2}$ are orthogonally additive. If $f$ is surjective, then it is additive.

It is easy to observe that neither the orthogonal additivity of $f$ implies the orthogonal additivity of $f^{2}$, nor the converse.

So far we have been considering orthogonalities which were homogenous. One can ask what is the form of orthogonally additive mappings defined on a normed space with isosceles orthogonality, Pythagorean orthogonality and norm derivative orthogonality.

Some partial answers were given by Szabó [180,181].

Theorem 2.13. Suppose that $(X,\|\cdot\|)$ is a real normed linear space with $\operatorname{dim} X \geq 3$ and $(Y,+)$ is an Abelian group. If a mapping $f: X \rightarrow Y$ is odd and satisfies (2.1) with isosceles orthogonality, then it is additive.

Theorem 2.14. (Characterization of i.p.s.) There exists a nontrivial, even, isosceles orthogonally additive mapping from a normed space $(X,\|\cdot\|), \operatorname{dim} X \geq$ 2 , to an Abelian group $(Y,+)$ if and only if $X$ is an inner product space.

Problem 2.1. What is the form of odd isosceles orthogonally additive mappings for a space $X$ with $\operatorname{dim} X=2$ ?

It seems that the Pythagorean orthogonality is the most difficult one for this kind of investigations.

Theorem 2.15. (Szabó [183]) Suppose that $(X,\|\cdot\|)$ is a real normed linear space with $\operatorname{dim} X \geq 3$ and $(Y,+)$ is an Abelian group. If $f: X \rightarrow Y$ is an odd mapping satisfying (2.1) with Pythagorean orthogonality, then it is additive.

Problem 2.2. (a) Determine even Pythagorean orthogonally additive mappings. (b) Determine odd Pythagorean orthogonally additive mappings in the case $\operatorname{dim} X=2$.

Of course in a normed space with Pythagorean orthogonality we cannot expect a similar result to Theorem 2.14 since in all such spaces, for arbitrarily fixed $z_{0} \in X \backslash\{0\}$ the function $f(x):=\|x\|^{2} z_{0}, x \in X$, is nonzero, even and orthogonally additive.

Looking for the form of solutions of the conditional equation

$$
f(x+y)=f(x)+f(y) \text { for all } x, y \in X \text { with } x \perp_{\rho} y
$$

we proved (see Alsina et al. $[6,7]$ ) that a real normed space $X$ with dimension at least 2 with $\rho$-orthogonality is in fact an orthogonality space in the sense of Rätz [149], and so, we know the form of solutions. As a by-product we obtained an alternative proof for the fact that a real norm space with Birkhoff orthogonality is an example of an orthogonality space (cf. Szabó $[177,179]$ ).

In [153], Rätz showed still another analogous characterization of inner product spaces: 
Theorem 2.16. (Characterization of i.p.s.) Let $(X,\|\cdot\|)$ be a real normed linear space, $\operatorname{dim} X \geq 2$, and let $\perp_{D}$ stand for the Diminnie orthogonality on $X$. Then $(X,\|\cdot\|)$ is an inner product space if and only if not every solution $f: X \rightarrow \mathbb{R}$ of the functional equation

$$
f(x+y)=f(x)+f(y) \quad \text { for all } x, y \in X \text { with } x \perp_{D} y
$$

is additive.

Instead of considering orthogonally additive mappings defined on the whole space we may study such conditional forms on a more restricted domain, for example on balls (see, e.g., Sikorska $[166,167]$ ).

Another direction of studies is to generalize the classical James orthogonality by defining the orthogonality relation as follows: $x \perp y$ if and only if $\varphi(x+y)=\varphi(x-y)$, where $\varphi$ is a given function with some properties (cf. Ger and Sikorska [90, Theorem 5], and Sikorska [169]). Of course, in case $\varphi=\|\cdot\|$, we have the isosceles orthogonality.

Let now $\mathcal{A}$ be a $C^{*}$-algebra and let $(X,\langle\cdot \mid \cdot\rangle)$ be a Hilbert $C^{*}$-module over $\mathcal{A}$.

Obviously, if $a$ is an additive mapping on $X$ and $b$ is an additive mapping on $\mathcal{A}$, then the mapping $f$ defined by

$$
f(x)=a(x)+b(\langle x \mid x\rangle) \text { for all } x \in X,
$$

is orthogonally additive.

During the 15th ICFEI (May 2013, Ustron, Poland), Ilišević presented results obtained jointly with Turnšek and Yang giving the conditions when the converse is true.

It is worth mentioning that the form $a+q$ of orthogonally additive functions, where $a$ is additive and $q$ is quadratic, is not always achieved.

Rätz [152] studied orthogonally additive mappings on free $\mathbb{Z}$-modules. In the case $\operatorname{dim}_{\mathbb{Z}} X=2$, he showed a deviation from the described situation, e.g., in the inner product space case (see also Rätz [156], Kuczma [120]).

At the end of this section we want to point out that also an additive setfunction $f: \mathfrak{M} \rightarrow \mathbb{R}$ defined on an algebra $\mathfrak{M}$, i.e., the function which satisfies a conditional equation

$$
f(A \cup B)=f(A)+f(B) \text { for all } A, B \in \mathfrak{M} \text { with } A \cap B=\emptyset
$$

is in fact an example of an orthogonally additive mapping.

2.1.2. Applications. We have already mentioned some applications of orthogonal additivity in mathematics. Namely, with its help we can give several characterizations of inner product spaces among normed spaces as well as of Hilbert spaces among Banach spaces.

The equation of orthogonal additivity can also give rise to some other mathematical problems (see, e.g., Maksa et al. [125] or Matkowski [128]). For other 
fields of mathematics where some kind of orthogonal additivity appears see Rätz [156].

There are quite interesting interactions of orthogonal additivity outside mathematics.

Equation (2.1) has its applications in physics, in the theory of ideal gas (see Aczél and Dhombres [1], Truesdell and Muncaster [188], Arkeryd and Cercignani [10]). In the three-dimensional Euclidean space, by means of (2.1) we obtain the formula for the distribution law of velocities in an ideal gas at a fixed temperature. Since for physical reasons, it is generally assumed that the distribution function is continuous, positive and even, the so called MaxwellBoltzmann distribution law has the form

$$
H(v)=A \exp \left(-a\|v\|^{2}\right)
$$

where $a, A$ are some positive constants, and the formula $a=\frac{m}{2 k T}$ connects $a$ with the mass of a molecule $m$, absolute temperature $T$ and the Boltzmann constant $k$ (cf. also the Boltzmann-Gronwall Theorem on summational invariants; [188]). In 1860, Maxwell obtained the above mentioned formula using another approach but also solving a functional equation, however, he made stronger assumptions (see [188]).

Equation (2.1) has got its applications also in actuarial mathematics in a premium calculation principle. It is shown (see Heijnen and Goovaerts [97]), that the variance principle is the only covariance-additive premium principle, i.e., satisfies the condition

$$
\pi(x+y)=\pi(x)+\pi(y) \quad \text { for all risks } x, y \text { with } \operatorname{cov}(x, y)=0 .
$$

\subsection{Jensen functional equation}

It is easy to see that a function which satisfies the orthogonal form of the Jensen functional equation

$$
f\left(\frac{x+y}{2}\right)=\frac{f(x)+f(y)}{2} \text { for all } x, y \in X \text { with } x \perp y
$$

between an orthogonality space $X$ and an Abelian group divisible by 2 is of the form $f(x)=h(x)+c$, where $h$ is orthogonally additive and $c$ is a real constant.

However, if one knows that $h$ is of the form $a+q$, where $a$ is additive and $q$ is quadratic, the immediate consequence is that the solutions of this conditional Jensen equation are unconditionally Jensen ones (see Ger [86]).

This nice fact we obtain thanks to the equality $f\left(\frac{x}{2}\right)=\frac{f(x)}{2}$ valid for all $x \in X$, which is in the sequel, a consequence of the relation $x \perp 0$ for all $x \in X$. This fact implies that while studying this conditional equation we are 
closer to investigations used for studies of unconditional forms rather than their conditional analogues.

A modified version of the Jensen equality was presented by Szostok [185]. A starting point for his considerations was the inequality

$$
f\left(\frac{x+y}{2}\right) \leq \gamma[f(x)+f(y)]
$$

postulated for some constant $\gamma \in\left(0, \frac{1}{2}\right)$ (see Kolwicz and Płuciennik [118]), having its background in Orlicz spaces. Namely, Szostok was studying the equality

$$
f\left(\frac{x+y}{2}\right)=\gamma\left(\frac{\|x-y\|}{\|x+y\|}\right)[f(x)+f(y)] \text { for all } x, y \text { with } x \neq-y,
$$

where $f$ maps a real normed linear space into the space of reals, which led in the sequel to an orthogonal Jensen equation (2.6) with the isosceles orthogonality.

These investigations gave rise to the studies of a generalized Cauchy equation (see Szostok $[186,187]$ ).

\subsection{Quadratic functional equation}

The studies of orthogonally quadratic functional equations, that is, conditional equations of the form

$$
f(x+y)+f(x-y)=2 f(x)+2 f(y) \text { for all } x, y \in X \text { with } x \perp y,
$$

started in 1966 with the paper by Vajzović [193], who described the form of continuous orthogonally quadratic functionals on a Hilbert space of dimension at least 3. More exactly, he proved that if $f: X \rightarrow \mathbb{K}$, where $(X,\langle\cdot \mid \cdot\rangle)$ is a real or complex Hilbert space, $\operatorname{dim} X \geq 3$, and $\mathbb{K} \in\{\mathbb{R}, \mathbb{C}\}$, satisfies the condition

$$
f(x+y)+f(x-y)=2 f(x)+2 f(y) \text { for all } x, y \in X \text { with }\langle x \mid y\rangle=0,
$$

then there exist a continuous linear operator $B$ and continuous quasi-linear operators $C$ and $D(C$ is quasi-linear if $C(x+y)=C(x)+C(y)$ and $C(\lambda x)=$ $\bar{\lambda} C(x)$ for all $x, y \in X$ and $\lambda \in \mathbb{C}$ ) such that

$$
f(x)=\langle B x \mid x\rangle+\langle C x \mid x\rangle+\langle x \mid D x\rangle \text { for all } x \in X .
$$

His result was generalized in 1986 by Drljević to $A$-orthogonality on a real (or complex) Hilbert space (see [63]). Namely, Drljević was considering (2.7) with $\langle x \mid y\rangle=0$ replaced by $\langle x \mid A y\rangle=0$ (we say that $x$ is $A$-orthogonal to $y$ ), where $A: X \rightarrow X$ is a continuous selfadjoint operator with $\operatorname{dim} A(X)>3$. Looking for the general continuous solution, he obtained the same form of $f$ as in (2.8). 
In the same year Fochi [70] showed that in inner product spaces of dimension not less than 3 , each real valued orthogonally quadratic mapping is unconditionally quadratic. In fact, this result remains true for values in a uniquely 2-divisible Abelian group.

In [71], Fochi proved even more, namely, she showed that both for real and complex valued functions, if $\operatorname{dim} A(X)>2$, then the solutions of the conditional $A$-orthogonal quadratic equation have to be quadratic (unconditionally).

Szabó [178] generalized the above mentioned results to a symmetric orthogonality introduced by a sesquilinear form on a linear space and for arbitrary mappings with values in an Abelian group. Before stating the main result given by Szabó, we introduce some notation.

Let $\Phi$ be a field such that $\operatorname{char} \Phi \notin\{2,3,5\}$, let $X$ be a vector space over $\Phi$ with $\operatorname{dim}_{\Phi} X \geq 3$ and let $(Y,+)$ be a 6 -torsion-free Abelian group, i.e., multiplication by 6 in $Y$ is injective. Furthermore, let $\varphi: X^{2} \rightarrow \Phi$ be a sesquilinear functional with respect to an automorphism $\xi: \Phi \rightarrow \Phi$, i.e., $\varphi$ is biadditive and $\varphi(\alpha x, \beta y)=\alpha \xi(\beta) \varphi(x, y)$ for all $x, y \in X$ and $\alpha, \beta \in \Phi$. Then define the $\varphi$-orthogonality relation $\perp^{\varphi}$ on $X$ by

$$
x \perp^{\varphi} y \quad \text { if and only if } \varphi(x, y)=0 .
$$

A vector $z \in X$ is said to be isotropic if $\varphi(z, z)=0$. For $x \in X$ define a linear functional $\varphi_{x}: X \rightarrow \Phi$ by $\varphi_{x}(t)=\varphi(t, x)$. Let us denote $X_{\varphi}^{*}=\left\{\varphi_{x}: x \in X\right\}$ which is a linear subspace of the algebraic conjugate space $X^{*}$ of $X$.

Theorem 2.17. Suppose that the $\varphi$-orthogonality on $X$ is symmetric, $\operatorname{dim} X_{\varphi}^{*} \geq$ 3 , and there exists a non-isotropic vector in $X$. If $Y$ is 6-torsion-free, then every $\varphi$-orthogonally quadratic mapping from $X$ to $Y$ is quadratic.

The problem of determining all solutions of the orthogonally quadratic functional equation on an arbitrary orthogonality space, or in a normed space with, e.g., Birkhoff, isosceles or Pythagorean orthogonalities remains open.

Some partial results were presented by Szabó during his lectures at the 5th International Conference on Functional Equations and Inequalities and on the 33rd International Symposium on Functional Equations in 1995 (see [184] and [182], respectively). However, we do not know his proofs, since the results were not published. We cite here the main theorems presented then.

Theorem 2.18. (Szabó, 5 ICFEI, 1995) Assume that $(X,\|\cdot\|)$ is a real normed space equipped with the Birkhoff orthogonality and $(Y,+)$ is an Abelian group. If $\operatorname{dim} X \geq 5$ and the norm is Gateaux differentiable, then $2 f$ is unconditionally quadratic whenever $f: X \rightarrow Y$ is a Birkhoff orthogonally quadratic mapping.

Theorem 2.19. (Szabó, 33 ISFE, 1995) If $(X,\|\cdot\|)$ is a strictly convex real normed space such that $\operatorname{dim} X \geq 4$ and $(Y,+)$ is an Abelian group, then for any isosceles orthogonally quadratic mapping $f: X \rightarrow Y, 2 f$ is unconditionally quadratic. 
So, let us collect now the still open problems.

Problem 2.3. Prove the theorems by Szabó. Are all assumptions necessary?

Problem 2.4. Determine the solutions of the orthogonally quadratic equation in an orthogonality space.

Problem 2.5. Determine the solutions of the orthogonally quadratic equation in a normed space with Pythagorean orthogonality.

In [76], Fochi was looking for the solutions of some pexiderized forms of an orthogonally quadratic equation, namely

$$
f(x+y)+f(x-y)=2 g(x)+2 h(y) \quad \text { for all } x, y \in X \text { with } x \perp y
$$

and

$$
f(x+y)+g(x-y)=h(x)+k(y) \quad \text { for all } x, y \in X \text { with } x \perp y .
$$

In an orthogonality space $X$ in the sense of Rätz (see Sect. 1.1.8) with a symmetric relation of orthogonality $\perp$ she proved the following

Theorem 2.20. The general solution $f, g, h: X \rightarrow \mathbb{R}$ of the functional equation (2.9) is given by

$$
\left\{\begin{array}{l}
f(x)=A(x)+Q(x)+f(0) \\
g(x)=A(x)+Q(x)+g(0) \\
h(x)=Q(x)+h(0),
\end{array}\right.
$$

for all $x \in X$, where $A: X \rightarrow \mathbb{R}$ is an additive function and $Q: X \rightarrow \mathbb{R}$ is orthogonally quadratic.

The final problem, however, stays unsolved. We do not know the general form of an orthogonally quadratic function.

For the next result assume that $(X,\langle\cdot \mid \cdot\rangle)$ is an inner product space, $\operatorname{dim} X>$ 2 .

Theorem 2.21. (Fochi [76]) Let $f, g, h, k: X \rightarrow \mathbb{R}$ be real functionals satisfying (2.10). Then there exist additive functions $A, B: X \rightarrow \mathbb{R}$, a quadratic function $Q: X \rightarrow \mathbb{R}$ and a function $\varphi:[0, \infty) \rightarrow \mathbb{R}$ such that for all $x \in X$,

$$
\left\{\begin{array}{l}
f(x)=\frac{1}{2}[A(x)+B(x)+Q(x)+\varphi(\|x\|)+h(0)+k(0)], \\
g(x)=\frac{1}{2}[A(x)-B(x)+Q(x)-\varphi(\|x\|)+h(0)+k(0)], \\
h(x)=A(x)+Q(x)+h(0), \\
k(x)=B(x)+Q(x)+k(0) .
\end{array}\right.
$$

Conversely, the above functionals satisfy (2.10). 


\subsection{Exponential functional equation}

Assume that $(X,\langle\cdot \mid \cdot\rangle)$ is an inner product space of dimension at least 2 . Some immediate consequences of Theorem 2.4 concerning the solutions $f: X \rightarrow \mathbb{R}$ of the conditional equation

$$
f(x+y)=f(x) f(y) \quad \text { for all } x, y \in X \text { with } x \perp y
$$

can be found, e.g., in Fochi [70].

We cite here two results from Baron and Rätz [22] and Baron and Forti [19], respectively.

Theorem 2.22. Let $T$ be a unit circle in $\mathbb{C}$. Suppose that a function $f: X \rightarrow T$ satisfies (2.11). If $f$ is continuous at the origin, then there exist a real constant $c$ and a continuous linear functional $g: X \rightarrow \mathbb{R}$ such that

$$
f(x)=e^{i\left(c\|x\|^{2}+g(x)\right)} \quad \text { for all } x \in X .
$$

Theorem 2.23. Suppose that a function $f: X \rightarrow \mathbb{C}$ satisfies (2.11). If there exist a neighbourhood $U \subset X$ of the origin and a positive real constant $\beta$ such that

$$
|f(x)| \leq \beta \Re(f(x))
$$

for every $x \in U$, then either $f$ vanishes on $X$, or

$$
f(x)= \begin{cases}0 & \text { if } x \in X \backslash\{0\} \\ 1 & \text { if } x=0\end{cases}
$$

or there exist additive functions $a: \mathbb{R} \rightarrow \mathbb{R}$ and $A: X \rightarrow \mathbb{R}$, a real constant $c$ and a continuous linear functional $g: X \rightarrow \mathbb{R}$ such that

$$
f(x)=\exp \left(a\left(\|x\|^{2}\right)+A(x)+i\left(c\|x\|^{2}+g(x)\right)\right) \quad \text { for all } x \in X .
$$

Before stating the next result we will recall some notion. Namely, we say that $f: X \rightarrow \mathbb{C}$ is measurable on rays if and only if for every $x \in X$ the function $t \mapsto f(t x), t \in \mathbb{R}$, is Lebesgue or Baire measurable.

Baron et al. in [20] were studying solutions $f: X \rightarrow \mathbb{C}$ of (2.11), different from zero at every point, assuming that the function $x \mapsto \frac{f(x)}{|f(x)|}, x \in X$, is continuous at the origin or measurable on rays. As a result they obtained among others the following.

Theorem 2.24. Suppose $f: X \rightarrow \mathbb{C}$ satisfies (2.11). Then either $f$ vanishes on $X \backslash\{0\}$ or $f(x) \neq 0$ for every $x \in X$. Moreover,

(i) if $f$ is continuous at the origin, then either $f$ vanishes on $X$ or there exist a complex constant $c$ and an $\mathbb{R}$-linear and continuous $g: X \rightarrow \mathbb{C}$ such that

$$
f(x)=e^{c\|x\|^{2}+g(x)} \quad \text { for all } x \in X
$$


(ii) if $f$ is measurable on rays, then either $f$ vanishes on $X$ or has the form (2.13), or $f$ has the form (2.15) with some complex constant $c$ and an $\mathbb{R}$-linear function $g: X \rightarrow \mathbb{C}$.

Later on, still with the assumption that $X$ is an inner product space, the studies were going in two directions.

First, instead of having (2.12) on a neighbourhood of zero it can be assumed that it is valid on a Christensen measurable set which is not a Christensen zero set (and $\beta=2$ ) on a Polish space, or it is valid on a second category set with the Baire property (and $\beta=2$ ), or it is valid on a set that has an algebraically interior point ${ }^{2}$. In each case we derive that the solution $f$ has to have the form (2.14) with some uniquely determined additive functions $a: \mathbb{R} \rightarrow \mathbb{R}$ and $A: X \rightarrow \mathbb{R}$, a linear function $g: X \rightarrow \mathbb{R}$, and a real constant $c$ (see Brzdęk [30, Corollary 5]).

The second approach is in the spirit of Theorem 2.24 (see Brzdęk [31, Theorem 3]).

Theorem 2.25. Assume that $f: X \rightarrow \mathbb{C}$ satisfies (2.11) and suppose that one of the conditions is valid:

(i) $X$ is a Polish space and $f$ is Christensen measurable;

(ii) $X$ is a Baire space and $f$ is Baire measurable;

(iii) $f$ is continuous at a point.

Then either $f$ vanishes on $X$, or $f$ is of the form (2.13), or $f$ has the form (2.15) with some $c \in \mathbb{C}$ and a continuous $\mathbb{R}$-linear functional $g: X \rightarrow \mathbb{C}$.

Another result under some measurability assumptions can be found in Brzdęk [33, Corollary 3].

We may also generalize the domain. Instead of an inner product space, we may consider an orthogonality space. We cite here one of the results from Brzdęk [32].

Theorem 2.26. Let $(X, \perp)$ be an orthogonality space and $f: X \rightarrow \mathbb{C}$ be a nonzero solution of (2.11), hemicontinuous at the origin. Then there exist $c \in \mathbb{C}$, unique linear functionals $a_{1}, a_{2}: X \rightarrow \mathbb{R}$, and a symmetric bilinear functional $L: X^{2} \rightarrow \mathbb{R}$, unique up to a multiplicative constant, such that $L(x, y)=0$ whenever $x \perp y$ and

$$
f(x)=\exp \left(a_{1}(x)+i a_{2}(x)+c L(x, x)\right) \text { for all } x \in X .
$$

Instead of an orthogonality space in the domain we may also consider a normed space with the isosceles James orthogonality. Brzdęk [34] proved the following.

\footnotetext{
${ }^{2}$ In a real linear space $X$ we say that $x \in X$ is an algebraically interior point of a set $D \subset X$ provided that for every $z \in X \backslash\{0\}$, there is $c_{z} \in \mathbb{R}, c_{z}>0$ such that $x+c z \in D$ for every $c \in\left(-c_{z}, c_{z}\right)$.
} 
Theorem 2.27. Let $X$ be a real normed linear space which is not an inner product space, $\operatorname{dim} X \geq 3$, and let $(S, \cdot)$ be a commutative semigroup with a neutral element. Suppose $f: X \rightarrow S$ satisfies (2.11) and there exists a nonzero element $x_{0}$ in $X$ such that $f\left(x_{0}\right)$ is invertible in $S$. Then $f$ is (unconditionally) exponential.

A simple consequence of the above is the fact that if we have a (commutative) field in the domain, then each orthogonally exponential mapping is (unconditionally) exponential.

\subsection{Other classical equations}

2.5.1. D'Alembert equation. It is interesting to compare the families of solutions of the functional equation characterizing the cosine function with its correspondent equation postulated only for orthogonal vectors (see Fochi [72]).

We restrict ourselves to inner product spaces $(X,\langle\cdot \mid \cdot\rangle)$ with dimension not less then 2 .

It is easy to see that the function $f: X \rightarrow \mathbb{R}$,

$$
f(x)=e^{h\left(\|x\|^{2}\right)} \quad \text { for all } x \in X,
$$

where $h: \mathbb{R} \rightarrow \mathbb{R}$ is an arbitrary additive function, is a solution of

$$
f(x+y)+f(x-y)=2 f(x) f(y) \text { for all } x, y \in X \text { with } x \perp y,
$$

but it is not necessarily a solution of

$$
f(x+y)+f(x-y)=2 f(x) f(y) \quad \text { for all } x, y \in X .
$$

So, the class of solutions of the functional equation (2.16) is a proper subset of the class of solutions of (2.17). Therefore, in order to characterize in the class of solutions of (2.16) the functions $f$ which satisfy (2.17), it is reasonable to add a suitable condition on $f$.

Theorem 2.28. (Fochi [72]) A non identically zero functional $f: X \rightarrow \mathbb{R}$ is a solution of (2.17) if and only if $f$ satisfies (2.16) and

$$
f(2 x)=2 f(x)^{2}-1 \quad \text { for all } x \in X .
$$

In [73], Fochi was dealing with some other classical trigonometric functional equations related to (2.16), namely

$$
f(x+y) f(x-y)=f(x)^{2}+f(y)^{2} \quad \text { for all } x, y \in X \text { with } x \perp y
$$

and

$$
f(x+y) f(x-y)=f(x)^{2}+f(y)^{2}-1 \quad \text { for all } x, y \in X \text { with } x \perp y .
$$


2.5.2. Alternative Cauchy equation. Next to the Cauchy functional equation we may consider its alternative form, namely

$$
f(x+y)^{2}=[f(x)+f(y)]^{2}
$$

or, more generally,

$$
\|f(x+y)\|=\|f(x)+f(y)\| .
$$

Similarly as in previous cases, we will study the conditional equation

$$
f(x+y)^{2}=[f(x)+f(y)]^{2} \text { for all } x, y \in X \text { with } x \perp y .
$$

One can see (cf. Fochi [74]) that already in inner product spaces the solutions of (2.19) differ from solutions of the orthogonal additivity equation (2.1).

Example 2.3. Let $h: \mathbb{R} \rightarrow \mathbb{R}$ be a given non-trivial additive function and $x_{0} \in X \backslash\{0\}$ be a fixed vector. For every $x \in X$ define

$$
f(x)=\left\{\begin{aligned}
h\left(\left\langle x \mid x_{0}\right\rangle\right) & \text { if } x=\lambda x_{0}, \lambda \in \mathbb{R}, \\
-h\left(\left\langle x \mid x_{0}\right\rangle\right) & \text { if } x \neq \lambda x_{0}, \lambda \in \mathbb{R} .
\end{aligned}\right.
$$

Then $f$ satisfies (2.19), but it is not orthogonally additive.

First, we present results describing separately the odd and the even solutions of (2.19).

Theorem 2.29. (Fochi [74]) Let $X$ be an inner product space with $\operatorname{dim} X \geq 3$ and let $f: X \rightarrow \mathbb{R}$ be a solution of the functional equation (2.19).

(i) If $f$ is odd, then there exists an additive mapping $h: X \rightarrow \mathbb{R}$ such that $|f(x)|=|h(x)|$ for all $x \in X$.

(ii) If $f$ is even, then $f$ is orthogonally additive, i.e., there exists an additive mapping $\varphi: \mathbb{R} \rightarrow \mathbb{R}$ such that $f(x)=\varphi\left(\|x\|^{2}\right)$ for all $x \in X$.

With some additional assumption on $f$ we have the following result.

Theorem 2.30. (Fochi [74]) Let $X$ be an inner product space with $\operatorname{dim} X \geq 3$ and let $f: X \rightarrow \mathbb{R}$ vanish only at zero. Then $f$ satisfies (2.19) if and only if, $f$ is orthogonally additive.

Similar investigations concerning the orthogonal forms of functional equations are done in Fochi's paper [75], where the author considers the conditional equation

$$
[f(x+y)-f(x)]^{2}=f(y)^{2} \quad \text { for all } x, y \in X \text { with } x \perp y .
$$


2.5.3. Cocycle equation. In 1998, Sikorska [165] asked about solutions of the conditional cocycle equation

$$
\begin{gathered}
F(x+y, z)+F(x, y)=F(x, y+z)+F(y, z) \text { for all } x, y, z \in X \\
\text { with } x \perp y, y \perp z, z \perp x .
\end{gathered}
$$

For a non-conditional case of the equation we have the following

Theorem. (Davison and Ebanks [52]) Let $M$ be a cancellative Abelian monoid and let $G$ be a divisible Abelian group. Then for every symmetric solution $F: M^{2} \rightarrow G$ of the equation

$$
F(x+y, z)+F(x, y)=F(x, y+z)+F(y, z) \quad \text { for all } x, y, z \in M
$$

there exists a function $f: M \rightarrow G$ such that

$$
F(x, y)=f(x)+f(y)-f(x+y) \quad \text { for all } x, y \in M
$$

Hypothesis. Let $(X,\langle\cdot \mid \cdot\rangle)$ be a real inner product space, $\operatorname{dim} X \geq 3$, and let $(G,+)$ be a divisible Abelian group (for example $G=\mathbb{R}$ ).

Then for every symmetric solution $F: X^{2} \rightarrow G$ of the conditional functional equation (2.20) there exists a function $f: X \rightarrow G$ such that

$$
F(x, y)=f(x)+f(y)-f(x+y) \text { for all } x, y \in X \text { with } x \perp y .
$$

The assumption $\operatorname{dim} X \geq 3$ in the Hypothesis allows us to avoid the necessity of using zero vectors. Rätz $[154,155]$ showed that just in these trivial cases we already have some variety. Namely, if $\operatorname{dim} X \leq 1$, the function $F$ has to be a Cauchy difference, while if $\operatorname{dim} X=2$, it is not the case.

Problem 2.6. Prove or disprove the above Hypothesis.

It is worth pointing out that an interesting and fruitful approach for arithmetic functions satisfying a conditional cocycle equation was done by Kochanek in [111, Lemma 2.2].

\subsection{Arithmetic functions}

As already observed by Rätz [156], there are connections between Pinsker theory, as sometimes the considered theory is called, and additive numbertheoretical (arithmetic) functions. Consider for functions $f: \mathbb{N} \rightarrow \mathbb{R}$ the conditional functional equation

$$
f(m n)=f(m)+f(n) \quad \text { for all } m, n \in \mathbb{N} \text { with } m \perp_{\mathrm{rp}} n,
$$

where in this case the orthogonality sign $\perp_{\mathrm{rp}}$ means that two elements are relatively prime, i.e., $(m, n)=1$.

Several mathematicians were looking for conditions which force an arithmetic additive function to be of the form $c \log n$. The first two results of this 
type, due to Erdös [65], assert that it is the case if $f$ satisfies one of the following conditions:

(i) $f$ is monotone,

(ii) $\lim _{x \rightarrow \infty}(f(x+1)-f(x))=0$.

Rényi in [158] gave a simplified and elegant proof of the Erdös theorem in the case (ii). Later, Kátai [108] and Máté [127] strengthened the assertion, assuming

(iii) $\liminf _{x \rightarrow \infty}(f(x+1)-f(x)) \geq 0$,

instead of (ii).

Schoenberg [162] extended (2.21) to the form

$$
f(x y)=f(x)+f(y) \text { for all } x, y \in \mathbb{Q}_{+} \text {with } x \perp_{\text {rp }} y
$$

for functions $f: \mathbb{Q}_{+} \rightarrow \mathbb{R}$, where the relation $x \perp_{\mathrm{rp}} y$ is understood as follows: if $x=\frac{m}{n}$ and $y=\frac{k}{l}$, for some positive integers $m, n, k, l$, are two reduced fractions, we say that $x$ and $y$ are relatively prime if $(m, k)=(m, l)=(n, k)=$ $(n, l)=1$. Schoenberg proves that solutions of (2.22) are in fact additive on the set of positive rationals.

\section{Stability of functional equations postulated for orthogonal vectors}

\subsection{Cauchy functional equation}

3.1.1. Hyers-Ulam stability. The origin of the stability problem traces back to Ulam (see $[191,192]$ ), who in 1940 asked to give conditions for the existence of a linear mapping near an approximately linear one. If $f$ is a function from a normed linear space $(X,\|\cdot\|)$ into a Banach space $(Y,\|\cdot\|)$ which satisfies with some $\varepsilon>0$ the inequality

$$
\|f(x+y)-f(x)-f(y)\| \leq \varepsilon \text { for all } x, y \in X,
$$

then Hyers [98] proved that there exists a unique additive mapping $a: X \rightarrow Y$ such that

$$
\|f(x)-a(x)\| \leq \varepsilon \quad \text { for all } x \in X .
$$

Moreover, if $\mathbb{R} \ni t \mapsto f(t x) \in Y$ is continuous for any fixed $x \in X$, then $a$ is linear (see also Rassias [146]). It should be mentioned that a version of Ulam's problem for real sequences appeared in the book of Pólya and Szegö [144].

Nowadays the theory of Hyers-Ulam stability is widely developed; one can consult, e.g., Forti [77], Hyers et al. [99], Jung [105], Moszner [137].

We start this section with citing a result by Ger and Sikorska [89, Theorem 1 and Remark 3] concerning the stability of the Cauchy functional equation postulated for orthogonal vectors in an orthogonality space. 
Theorem 3.1. Let $(X, \perp)$ be an orthogonality space. Given $\varepsilon \geq 0$ and a real Banach space $(Y,\|\cdot\|)$, let $f: X \rightarrow Y$ be a mapping such that

$$
\|f(x+y)-f(x)-f(y)\| \leq \varepsilon \quad \text { for all } x, y \in X \text { with } x \perp y .
$$

Then there exists exactly one orthogonally additive mapping $g: X \rightarrow Y$ such that

$$
\|f(x)-g(x)\| \leq \frac{16}{3} \varepsilon \quad \text { for all } x \in X .
$$

Actually, the norm structure in $Y$ may be avoided. We have (see Ger and Sikorska [89, Remark 4])

Theorem 3.2. Let $(X, \perp)$ be an orthogonality space and let $Y$ be a real sequentially complete Hausdorff linear topological space. Assume that a bounded convex and symmetric with respect to zero set $V \subset Y$ and a mapping $f: X \rightarrow Y$ are given such that

$$
f(x+y)-f(x)-f(y) \in V \quad \text { for all } x, y \in X \text { with } x \perp y .
$$

Then there exists exactly one orthogonally additive mapping $g: X \rightarrow Y$ such that

$$
f(x)-g(x) \in \frac{16}{3} \operatorname{seq} \operatorname{cl} V \quad \text { for all } x \in X .
$$

In fact, some stability results for $A$-orthogonal vectors in Hilbert spaces appeared already in the paper by Drljević and Mavar [64], but just [89] gave probably a rise to a huge number of papers considering various kinds of stability problems of various functional equations postulated for orthogonal vectors.

It is worth recalling that the orthogonally additive mapping appearing in the assertion of Theorems 3.1 and 3.2 is of the form $a+q$, where $a$ is additive and $q$ is quadratic (cf. Theorem 2.3).

Similar results can be obtained in case $(X,\|\cdot\|)$ is a real normed linear space with $\operatorname{dim} X \geq 2$ and with the James orthogonality relation on $X$ (see Sikorska [164] or [168]).

Problem 3.1. Is the orthogonal Cauchy functional equation stable if we consider as a domain a real normed linear space with the Pythagorean orthogonality?

In 2010, Fechner and Sikorska [69] published a generalization of the above results (see also Sikorska [173]). Also, the estimating constant was sharpened.

Theorem 3.3. Let $X$ be an Abelian group and let $\perp$ be a binary relation defined on $X$ with the properties:

( $\alpha$ ) if $x, y \in X$ and $x \perp y$, then we have $x \perp-y,-x \perp y$ and $2 x \perp 2 y$;

( $\beta$ ) for every $x \in X$, there exists $y \in X$ such that $x \perp y$ and $x+y \perp x-y$. 
Further, let $(Y,\|\cdot\|)$ be a (real or complex) Banach space. Given $\varepsilon \geq 0$, let $f: X \rightarrow Y$ be a mapping such that

$$
\|f(x+y)-f(x)-f(y)\| \leq \varepsilon \quad \text { for all } x, y \in X \text { with } x \perp y .
$$

Then there exists a mapping $g: X \rightarrow Y$ such that

$$
g(x+y)=g(x)+g(y) \quad \text { for all } x, y \in X \text { with } x \perp y,
$$

and

$$
\|f(x)-g(x)\| \leq 5 \varepsilon,
$$

for all $x \in 2 X=\{2 x: x \in X\}$. Moreover, the mapping $g$ is unique on the set $2 X$.

In case $X$ is uniquely 2-divisible, we get (3.4) on the whole group $X$; however, there are examples of non-trivial groups with $2 X=\{0\}$ for which our assertion does not bring much information.

Remark 3.1. The above results can be applied both in an orthogonality space and in a normed space with James orthogonality. However, the problem remains open in the case of Pythagorean orthogonality.

Along the results for functions with the domain being the whole space we may consider orthogonal vectors only from some set. We give some results where the role of this set is played by a ball (see Sikorska [166,167]).

We start with the results in an inner product space.

Theorem 3.4. Let $X$ be a real inner product space, $\operatorname{dim} X=N(N \geq 2), Y$ be a real sequentially complete linear topological space, and let $V$ be a nonempty bounded convex and symmetric with respect to zero subset of $Y$. Let, further, $B$ denote a ball in $X$ centered at the origin. If a function $f: B \rightarrow Y$ fulfils the condition

$$
f(x+y)-f(x)-f(y) \in V \quad \text { for all } x, y \in B \text { with } x+y \in B \text { and } x \perp y,
$$

then there exist additive functions $a: X \rightarrow Y, b: \mathbb{R} \rightarrow Y$ and a constant $k=k(N)$ such that

$$
f(x)-a(x)-b\left(\|x\|^{2}\right) \in k \operatorname{seq} c l \quad \text { for all } x \in B .
$$

Remark 3.2. It is easy to see that $g=a+b \circ\|\cdot\|^{2}$ is orthogonally additive but, in general, such $g$ is not uniquely determined.

Example 3.1. Let $X=\mathbb{R}^{2}$ with the Euclidean norm, $Y=\mathbb{R}, B=\{x \in X$ : $\|x\| \leq 1\}$ and let $f: B \rightarrow \mathbb{R}$ be an additive function. Obviously, for all vectors $x, y \in B$ such that $x+y \in B$ and $x \perp y$ we have

$$
|f(x+y)-f(x)-f(y)| \leq \varepsilon .
$$

Then both $g_{1}:=f$ and $g_{2}(x):=f(x)+c \cdot x, x \in \mathbb{R}^{2}$, with constant $c \in \mathbb{R}^{2}$ such that $\|c\|<k \varepsilon$, fulfil the condition 


$$
|f(x)-g(x)| \leq k \varepsilon \text { for all } x \in B
$$

(the sign "." stands here for the standard inner product in $\mathbb{R}^{2}$ ).

Assume now that the domain $(X,\|\cdot\|)$ is a real normed linear space with Birkhoff orthogonality, $\operatorname{dim} X=N \geq 2$, the target space $Y$ is a real sequentially complete linear topological space, and $V$ is a nonempty, bounded subset of $Y$ which is convex and symmetric with respect to zero.

Theorem 3.5. If $f: B \rightarrow Y$ fulfils the condition (3.5) with the Birkhoff orthogonality relation $\perp$, then there exist an additive function $a: X \rightarrow Y$, a quadratic function $q: X \rightarrow Y$ and a constant $K=K(N)$ such that

$$
f(x)-a(x)-q(x) \in K \operatorname{seq} \mathrm{cl} V \quad \text { for all } x \in \frac{1}{2} B .
$$

The above result is slightly weaker than expected. Apart from the fact that the approximating function $g:=a+q$ is not uniquely determined, it does not need to be orthogonally additive, it means that the quadratic part may fail to be orthogonally additive.

Example 3.2. Take $f: \mathbb{R}^{2} \rightarrow \mathbb{R}$ which is additive and assume that a norm $\|\cdot\|$ in $\mathbb{R}^{2}$ does not come from an inner product. Consider the Birkhoff orthogonality in $\mathbb{R}^{2}$. Then of course, $f$ satisfies (3.5).

Take an arbitrary $\varepsilon>0$ and define $g: \mathbb{R}^{2} \rightarrow \mathbb{R}$ as $g(x):=f(x)+c\left(x_{1}^{2}+x_{2}^{2}\right)$ for all $x=\left(x_{1}, x_{2}\right) \in \mathbb{R}^{2}$ and with a real constant $c$ such that $|c| \leq \frac{4 K \varepsilon}{\alpha}$, where $\alpha$ is a positive number such that for all $x \in \mathbb{R}^{2}$ we have $\left(x_{1}^{2}+x_{2}^{2}\right)^{\alpha} \leq \alpha\|x\|^{2}$ (since the Euclidean norm and $\|\cdot\|$ are equivalent). It is easy to show that $g-f$ is quadratic.

Moreover, for every $x \in \frac{1}{2} B$, where $B$ is a unit ball in $\mathbb{R}^{2}$, we have

$$
|f(x)-g(x)|=\left|c\left(x_{1}^{2}+x_{2}^{2}\right)\right|<K \varepsilon .
$$

However, the function $g$ is not orthogonally additive on the half-ball. To see this, take arbitrary $x=\left(x_{1}, x_{2}\right)$ and $y=\left(y_{1}, y_{2}\right)$ from $\frac{1}{2} B$ such that $x \perp y$ and note that

$$
g(x+y)-g(x)-g(y)=2 c\left(x_{1} y_{1}+x_{2} y_{2}\right) .
$$

The above difference cannot always be zero. Otherwise the orthogonality relation in the sense of Birkhoff would be equivalent to the orthogonality relation connected with some inner product defined on $\mathbb{R}^{2}$, which leads to a contradiction.

Assume now that $(X,\|\cdot\|)$ is a real uniformly convex space ${ }^{3}$ with Birkhoff orthogonality relation, $\operatorname{dim} X=N \geq 2$, and $Y, V$ and $B$ are the same as before. This time we get an approximation on the whole ball.

\footnotetext{
${ }^{3} \mathrm{~A}$ normed linear space $(X,\|\cdot\|)$ is called uniformly convex if and only if for every $\varepsilon>0$ there exists $\delta(\varepsilon)>0$ such that for all $x, y \in X$ if $\|x\|=\|y\|=1$ and $\|x-y\| \geq \varepsilon$ then $\left\|\frac{1}{2}(x+y)\right\|<1-\delta(\varepsilon)$.
} 
Theorem 3.6. If a function $f: B \rightarrow Y$ satisfies (3.5), then there exist an additive function $a: X \rightarrow Y$, a quadratic function $q: X \rightarrow Y$ and a constant $c=c(N)$ such that

$$
f(x)-a(x)-q(x) \in \operatorname{cseq} \mathrm{cl} V \quad \text { for all } \quad x \in B .
$$

3.1.2. Generalized stability. In what follows we consider the conditional inequality

$$
\|f(x+y)-f(x)-f(y)\| \leq \varphi(x, y) \quad \text { for all } x, y \in X \text { with } x \perp y
$$

with some function $\varphi: X \times X \rightarrow[0, \infty)$ (see Sikorska [168]; cf. also Găvruţa and Găvruţa [80]).

Let $(X, \perp)$ be an orthogonality space with $\lambda=1$ (see Sect. 1.1.8). Consider a function $\varphi: X \times X \rightarrow[0, \infty)$ such that

(a) for every $x \in X$ the series $\sum_{n=1}^{\infty} 2^{-n} \varphi\left(2^{n-1} x, 2^{n-1} x\right)$ is convergent or for every $x \in X$ the series $\sum_{n=1}^{\infty} 2^{n-1} \varphi\left(2^{-n} x, 2^{-n} x\right)$ is convergent; denote such a sum by $\Phi(x)$;

(b) for every $x \in X$ the series $\sum_{n=0}^{\infty} 4^{1-n} \varphi\left(2^{n-1} x, 2^{n-1} x\right)$ is convergent or for every $x \in X$ the series $\sum_{n=1}^{\infty} 4^{n} \varphi\left(2^{-n} x, 2^{-n} x\right)$ is convergent; denote such a sum by $\Psi(x)$;

(c) for all $x, y \in X$ such that $x \perp y$ we have

$$
\lim _{n \rightarrow \infty} 2^{-n} \varphi\left(2^{n} x, 2^{n} y\right)=0 \quad \text { or } \quad \lim _{n \rightarrow \infty} 2^{n} \varphi\left(2^{-n} x, 2^{-n} y\right)=0
$$

for respective cases from (a);

(d) for all $x, y \in X$ such that $x \perp y$ we have

$$
\lim _{n \rightarrow \infty} 4^{-n} \varphi\left(2^{n} x, 2^{n} y\right)=0 \quad \text { or } \quad \lim _{n \rightarrow \infty} 4^{n} \varphi\left(2^{-n} x, 2^{-n} y\right)=0
$$

for respective cases from (b);

(e) there exists $M>0$ such that for all $x, y \in X$, if $x \perp y$ and $x+y \perp x-y$ then

$$
\max \{\varphi( \pm x, \pm y), \varphi( \pm(x+y), \pm(x-y))\} \leq M \varphi(x, x) .
$$

Theorem 3.7. Let $(X, \perp)$ be an orthogonality space, $(Y,\|\cdot\|)$ be a real Banach space and let $\varphi: X \times X \rightarrow[0, \infty)$ satisfy conditions (a)-(e). If a function $f: X \rightarrow Y$ satisfies (3.6), then there exists exactly one orthogonally additive function $g: X \rightarrow Y$ such that

$$
\|f(x)-g(x)\| \leq M[3 \Phi(x)+\Psi(x)] \text { for all } x \in X .
$$

Remark 3.3. An analogous approach is used while studying the generalized orthogonal stability of the Jensen functional equation

$$
\left\|f\left(\frac{x+y}{2}\right)-\frac{f(x)+f(y)}{2}\right\| \leq \varphi(x, y) \text { for all } x, y \in X \text { with } x \perp y,
$$


or Pexider functional equation. For the latter one, see the beginning of the paper by Fechner and Sikorska [68]. For the former one we should have a function $\varphi$ with the properties

(a)' for every $x \in X$ the series $\sum_{n=1}^{\infty} 2^{1-n} \varphi\left(2^{n} x, 0\right)$ is convergent or for every $x \in X$ the series $\sum_{n=1}^{\infty} 2^{n} \varphi\left(2^{1-n} x, 0\right)$ is convergent; denote such a sum by $\Lambda(x)$;

and (c) with conditions corresponding to the respective cases from (a)'. We use here only (i) and (iii) with $\alpha=\beta$ from the properties of the orthogonality space.

Let $\bar{f}:=f-f(0)$. Then $\bar{f}(0)=0$ and by the assumptions we get the existence of an orthogonally additive mapping $a$ such that

$$
\|\bar{f}(x)-a(x)\| \leq \Lambda(x) \text { for all } x \in X .
$$

3.1.3. Some applications. By means of the results from the last section we may prove various kinds of "sandwich" theorems, where we separate orthogonally subadditive (3.7) and orthogonally superadditive (3.8) functions. We give here one example (cf. Fechner and Sikorska [68, Proposition 3]). More examples and more general forms of the theorem can be found in [68].

Theorem 3.8. Let $(X,\|\cdot\|)$ be a real normed space, $\operatorname{dim} X \geq 2$, with Birkhoff orthogonality. Assume that $p, q: X \rightarrow \mathbb{R}$ satisfy

$$
\begin{aligned}
& p(x+y) \leq p(x)+p(y) \quad \text { for all } x, y \in X \text { with } x \perp_{B} y, \\
& q(x+y) \geq q(x)+q(y) \quad \text { for all } x, y \in X \text { with } x \perp_{B} y
\end{aligned}
$$

and

$$
q(x) \leq p(x) \text { for all } x \in X .
$$

If $p(x)-q(x) \leq c\|x\|^{r}$ for all $x \in X$, where $c, r$ are positive constants and $r>2$, then there exists a unique orthogonally additive mapping $f: X \rightarrow \mathbb{R}$ such that with some positive constant $d$,

$$
q(x)-d\|x\|^{r} \leq f(x) \leq p(x)+d\|x\|^{r} \quad \text { for all } x \in X .
$$

With some additional assumptions imposed on the functions $p$ and $q$ we get the approximation $q \leq f \leq p$ in the above theorem (see [68, Theorem 3]).

Some other results on orthogonally superadditive functions can be found in Fechner [67].

3.1.4. Functional congruences. Let $X$ be a real linear space and let $\perp$ be an orthogonality relation defined in this space. Let $\mathcal{F}$ and $\mathcal{F}^{(2)}$ be classes of functions defined on $X$ and on $X^{2}$, respectively, and with values in a group $(Y,+)$. We say that the pair $\left(\mathcal{F}, \mathcal{F}^{(2)}\right)$ has the orthogonal double difference property if every function $f: X \rightarrow Y$ satisfying $f(x+y)-f(x)-f(y) \in \mathcal{F}^{(2)}$ for orthogonal vectors is of the form $f=g+A$, where $g \in \mathcal{F}$ and $A$ is orthogonally additive. 
In the classical stability problem the classes $\mathcal{F}$ and $\mathcal{F}^{(2)}$ are the classes of bounded functions. But similarly we can consider other pairs $\left(\mathcal{F}, \mathcal{F}^{(2)}\right)$, namely with integer valued functions or, more general, classes of functions with values in a given discrete subgroup.

The first result comes from papers by Baron and Rätz [22] and Baron [15].

Theorem 3.9. Let $X$ be a real inner product space with $\operatorname{dim} X \geq 2,(G,+)$ a topological Abelian group, and $K$ a discrete subgroup of $G$. If $f: X \rightarrow G$ fulfils the condition

$$
f(x+y)-f(x)-f(y) \in K \quad \text { for all } x, y \in X \text { with } x \perp y,
$$

and it is continuous at a point, then there exist continuous additive functions $a: \mathbb{R} \rightarrow G$ and $A: X \rightarrow G$ such that

$$
f(x)-a\left(\|x\|^{2}\right)-A(x) \in K \quad \text { for all } \quad x \in X .
$$

In fact the above result (see Baron [15]) was first proved by Baron and Rätz in [22] under the additional assumption that $G$ is continuously divisible by 2 (the function $u \mapsto 2 u$ is a homeomorphism of $G$ onto $G$ ), and $f$ is continuous at the origin. Brzdęk [31] generalized the result of Baron and Rätz [22] showing that $f$ can be supposed continuous at any point and that the assumption concerning $G$ can be replaced by a weaker one: $2 u \neq 0$ for $u \in G \backslash\{0\}$.

The representation obtained in the above theorem does not remain valid without a regularity condition. In order to see this we may consider a function $\varphi: \mathbb{R} \rightarrow \mathbb{R}$ such that $\varphi(s+t)-\varphi(s)-\varphi(t) \in \mathbb{Z}$ for all $s, t \in \mathbb{R}$, but for every additive function $a: \mathbb{R} \rightarrow \mathbb{R}$ there exists $t \in \mathbb{R}$ such that $\varphi(t)-a(t) \notin \mathbb{Z}$. The existence of such a function follows from Godini's paper [92, Example 2]. We use this $\varphi$ in the following two examples (see Baron and Rätz [22]).

Example 3.3. Define $f: \mathbb{R}^{2} \rightarrow \mathbb{R}$ by the formula $f\left(x_{1}, x_{2}\right)=\varphi\left(x_{1}\right)$. Then

$$
f(x+y)-f(x)-f(y) \in \mathbb{Z} \text { for all } x, y \in \mathbb{R}^{2}
$$

and one can show that there is no additive function $A: \mathbb{R}^{2} \rightarrow \mathbb{R}$ such that

$$
f(x)-A(x) \in \mathbb{Z} \text { for all } x \in \mathbb{R}^{2} .
$$

Example 3.4. Define $f: \mathbb{R}^{2} \rightarrow \mathbb{R}$ by the formula $f(x)=\varphi\left(\|x\|^{2}\right)$. Then

$$
f(x+y)-f(x)-f(y) \in \mathbb{Z} \text { for all } x, y \in \mathbb{R}^{2} \text { with } x \perp y,
$$

and one can show that there are no additive functions $a: \mathbb{R} \rightarrow \mathbb{R}$ and $A: \mathbb{R}^{2} \rightarrow$ $\mathbb{R}$ such that

$$
f(x)-a\left(\|x\|^{2}\right)-A(x) \in \mathbb{Z} \text { for all } x \in \mathbb{R}^{2} .
$$

A particular case where the target space is the space of reals and the discrete subgroup is the set of integers was examined first by Baron and Forti in [19]. 
Theorem 3.10. Let $X$ be a real inner product space with $\operatorname{dim} X \geq 2$. Suppose $f: X \rightarrow \mathbb{R}$ satisfies

$$
f(x+y)-f(x)-f(y) \in \mathbb{Z} \quad \text { for all } x, y \in X \text { with } x \perp y .
$$

If there exist a neighbourhood $U$ of the origin and $\gamma \in(0,1 / 4)$ such that $f(U) \subset$ $(-\gamma, \gamma)+\mathbb{Z}$, then there exist a real constant $c$ and a continuous linear functional $h: X \rightarrow \mathbb{R}$ such that

$$
f(x)-c\|x\|^{2}-h(x) \in \mathbb{Z} \quad \text { for all } x \in X .
$$

Brzdęk [30] showed that an analogous result can be obtained in the cases: $U$ is a set of the second category and with the Baire property, or $U$ is a Christensen measurable nonzero set, or $U$ has an algebraically interior point.

Theorem 3.11. Let $X$ be a real inner product space with $\operatorname{dim} X \geq 2$, $\gamma \in \mathbb{R}, \gamma>0, D \subset X$, and let $f: X \rightarrow \mathbb{R}$ be a functional satisfying (3.11) such that $f(D) \subset(-\gamma, \gamma)+\mathbb{Z}$. Suppose that one of the three following conditions is valid:

(i) $X$ is a Polish space, $D$ is a Christensen measurable set which is not a Christensen zero set, and $\gamma=\frac{1}{6}$;

(ii) $D$ is of the second category and with the Baire property and $\gamma=\frac{1}{6}$;

(iii) $D$ has an algebraically interior point and $\gamma<\frac{1}{4}$.

Then there exist a unique linear functional $h: X \rightarrow \mathbb{R}$ and a unique constant $c \in \mathbb{R}$ such that (3.12) is satisfied. Moreover, if (ii) holds then $h$ is continuous.

The following theorem describes the functions which are Christensen or Baire measurable and for which the Cauchy difference is in a discrete subgroup (Brzdęk [31]).

Theorem 3.12. Assume that $X$ is a real inner product space with $\operatorname{dim} X \geq 2$, $(G,+)$ is an Abelian topological group and $K$ a discrete subgroup of $G, x+x \neq 0$ for $x \in G, x \neq 0$. Let $f: X \rightarrow G$ be a function satisfying (3.9). If one of the conditions

(i) $X$ is a Polish space, $G$ is $\sigma$-bounded ${ }^{4}$ and $f$ is Christensen measurable;

(ii) $X$ is a Baire space

is satisfied, then there exist continuous additive functions $a: \mathbb{R} \rightarrow G$ and $A: X \rightarrow G$ such that (3.10) holds.

In all the results we were considering so far the domain was an inner product space. Of course, it is also possible to think of a linear space with an abstract orthogonality relation, or even of a group with orthogonality in the sense of Baron and Volkmann [24] (see Sect. 1.1.11).

\footnotetext{
${ }^{4}$ We say that a topological group $(G,+)$ is $\sigma$-bounded provided that, for every open neighbourhood $U \subset G$ of 0 there is a sequence $\left(x_{n}: n \in \mathbb{N}\right) \subset G$ with $H=\bigcup\left\{U+x_{n}: n \in \mathbb{N}\right\}$. For instance, every topological group $(G,+)$ possessing a dense countable subset is $\sigma$-bounded.
} 
In [33], Brzdęk studied universally, Christensen or Baire measurable functions defined on a real linear topological space with axiomatic orthogonality relation by Rätz, and with values in $\mathbb{C}$. In [198], Wyrobek-Kochanek proved the following result.

Theorem 3.13. Assume that $(G,+)$ is an Abelian topological group such that the mapping $u \mapsto 2 u, u \in G$, is a homeomorphism and the following condition holds: every neighbourhood of zero in $G$ contains a neighbourhood $U$ of zero such that $U \subset 2 U$ and $G=\bigcup\left\{2{ }^{n} U: n \in \mathbb{N}\right\}$. Assume that $\perp$ is an orthogonality relation in $G$ in the sense of Baron and Volkmann, $(H,+)$ is an Abelian topological group and $K$ is a discrete subgroup of $H$. Then a function $f: G \rightarrow H$ continuous at a point satisfies

$$
f(x+y)-f(x)-f(y) \in K \quad \text { for all } x, y \in G \text { with } x \perp y
$$

if, and only if, there exist a continuous additive function $a: G \rightarrow H$ and $a$ continuous biadditive and symmetric function $b: G \times G \rightarrow H$ such that

$$
f(x)-a(x)-b(x, x) \in K \quad \text { for all } x \in G
$$

and

$$
b(x, y)=0 \quad \text { for all } x, y \in G \text { with } x \perp y .
$$

Moreover, $a$ and $b$ are uniquely determined.

Theorem 3.13 generalizes earlier results from the paper by Baron and Kucia [21] and also Theorem 2.9 from [32] (where Brzdęk obtained the continuity of $q(x):=b(x, x), x \in X$, only at a point).

Assume that $G$ is a topological Abelian group, $\mathfrak{M}$ is a $\sigma$-algebra and $\mathfrak{I}$ is a proper $\sigma$-ideal of subsets of $G$ which fulfil the condition

$$
0 \in \operatorname{Int}(A-A) \text { if } A \in \mathfrak{M} \backslash \mathfrak{I} .
$$

Continuing the studies of Brzdęk from [33] (for functions from an orthogonality space to the complex field), Kochanek and Wyrobek [114], working now on groups with the orthogonality relation in the sense of Baron and Volkmann, faced a problem: under what assumptions does an $\mathfrak{M}$-measurable mapping $f$ from $(G,+)$ into an Abelian topological group $(H,+)$, which is orthogonally additive modulo $K$, a discrete subgroup of $H$, admit a factorization (3.14) with a continuous additive function $a: G \rightarrow H$ and a continuous biadditive function $b: G \times G \rightarrow H$ ?

Namely, they have obtained the following results.

Theorem 3.14. [114, Theorem 1] Assume that $H$ is a separable metric group,

(G1) the mapping $G \ni x \mapsto 2 x$ is a homeomorphism,

(G2) every neighbourhood of zero in $G$ contains a neighbourhood $U$ of zero such that $U \subset 2 U$ and $G=\bigcup\left\{2^{n} U: n \in \mathbb{N}\right\}$,

(G3) either $G$ is a first countable Baire group, or $G$ is metric separable, or $G$ is metric and $\mathfrak{M}$ contains all Borel subsets of $G$, 
(G4) $x \pm 2 A \in \mathfrak{M}$ for all $x \in G$ and $A \in \mathfrak{M}$.

Then an $\mathfrak{M}$-measurable function $f: G \rightarrow H$ satisfies (3.13) if and only if there exist a continuous additive function $a: G \rightarrow H$ and a continuous biadditive symmetric function $b: G \times G \rightarrow H$ such that the factorization (3.14) is valid and (3.15) is satisfied. Moreover, the functions a and $b$ are uniquely determined.

Corollary 3.1. [114, Corollary 1] Assume that $H$ is a separable metric group and (G1),(G2) hold. If either $G$ is a first countable Baire group and $f: G \rightarrow H$ is Baire measurable, or $G$ is a Polish group and $f: G \rightarrow H$ is Christensen measurable, then $f$ satisfies (3.13) if and only if there exist a continuous additive function $a: G \rightarrow H$ and a continuous biadditive symmetric function $b: G \times G \rightarrow H$ such that (3.14) and (3.15) hold. Moreover, the functions a and $b$ are uniquely determined.

Baire and Christensen measurable solutions of (3.13) were examined before by Brzdęk in [31] for the orthogonality given by an inner product (in inner product spaces) and in [33] for more abstract orthogonality in linear topological spaces.

Under weaker assumptions Kochanek and Wyrobek obtained the factorization (3.14) with separately continuous biadditive function only (as it is in Brzdęk [33, Theorem 1]).

Theorem 3.15. [114, Theorem 2] Assume (G1), (G2), (G4) and let $H$ be a separable metric group. Then an $\mathfrak{M}$-measurable function $f: G \rightarrow H$ satisfies (3.13) if and only if there exist a continuous additive function $a: G \rightarrow H$ and a biadditive symmetric function $b: G \times G \rightarrow H$, continuous in each variable, such that (3.14) and (3.15) hold. Moreover, the functions a and $b$ are uniquely determined.

Studying pexiderized forms of the Cauchy difference often brings interesting and surprising results (cf., e.g., Sikorska [170]). Pexiderized forms of (3.13) were investigated by Bajger [12] and Wyrobek-Kochanek [199].

3.1.5. Orthogonal additivity almost everywhere. Assume that $f$ is defined on the Euclidean space $X=\mathbb{R}^{n}$ and takes values in an Abelian group $(Y,+)$. Kochanek and Wyrobek-Kochanek [115] were studying the functions which satisfy (2.1) almost everywhere in a sense that

$$
f(x+y)=f(x)+f(y) \text { for all }(x, y) \in\left\{(x, y) \in X^{2}:\langle x \mid y\rangle=0\right\} \backslash Z,
$$

where $Z$ is a negligible subset of the $(2 n-1)$-dimensional manifold $\perp \subset \mathbb{R}^{2 n}$.

They have concluded that $f$ coincides almost everywhere with some orthogonally additive mapping.

Considerations of this type go back to a problem posed by Erdős [66], concerning the unconditional version of the Cauchy functional equation. It 
was solved by de Bruijn [29] and, independently, by Jurkat [106], and also generalized by Ger [83].

3.1.6. Nearly additive set functions. At the end of Sect. 2.1.1 we observed that additive set functions may be treated as examples of orthogonally additive mappings. Now we present some results concerning the stability of Eq. (2.5).

Theorem 3.16. (Kalton and Roberts [107]) Let $\mathfrak{M}$ be an algebra of sets and $f: \mathfrak{M} \rightarrow \mathbb{R}$ be a function satisfying

$$
|f(A \cup B)-f(A)-f(B)| \leq 1 \text { for all } A, B \in \mathfrak{M} \text { with } A \cap B=\emptyset .
$$

Then there exist a constant $C<45$ and an additive set-function $\mu: \mathfrak{M} \rightarrow \mathbb{R}$ such that

$$
|f(A)-\mu(A)| \leq C \quad \text { for all } A \in \mathfrak{M} .
$$

Pawlik [142] gave an example showing that $C \geq \frac{3}{2}$.

The above theorem was a motivation for Kochanek to study the stability problem for vector measures (understood as finitely additive set functions) (see [112]). He was investigating the properties of those Banach spaces which have the so called SVM (stability of vector measures) property; namely, we say that a Banach space $X$ has the SVM property if there exists a constant $v(X)<\infty$ (depending only on $X$ ) such that given any set algebra $\mathfrak{M}$ and any mapping $\nu: \mathfrak{M} \rightarrow X$ satisfying

$$
\|\nu(A \cup B)-\nu(A)-\nu(B)\| \leq 1 \text { for all } A, B \in \mathfrak{M} \text { with } A \cap B=\emptyset,
$$

there is a vector measure $\mu: \mathfrak{M} \rightarrow X$ such that

$$
\|\nu(A)-\mu(A)\| \leq v(X) \text { for all } A \in \mathfrak{M} .
$$

3.1.7. Arithmetic functions. A natural stability question for arithmetic additive functions may be formulated as (see Kochanek [109]): assume that for a fixed $\varepsilon \geq 0$ we have the conditional inequality

$$
|f(m n)-f(m)-f(n)| \leq \varepsilon \text { for all } m, n \in \mathbb{N} \text { with } m \perp_{\mathrm{rp}} n .
$$

Does it imply that $f$ is approximately equal to some arithmetic additive function, that is a function satisfying (2.21)? Of course the condition that $m$ and $n$ are relatively prime, appearing in (3.16), causes that the direct method using Cauchy sequences cannot be used.

Considering results of Erdős, Kátai, Máté (see [65,108,127]), Kochanek [109] proved the following.

Theorem 3.17. Assume that a function $f: \mathbb{N} \rightarrow \mathbb{R}$ satisfies (3.16) and

$$
\liminf _{n \rightarrow \infty}(f(n+1)-f(n)) \geq 0 .
$$

Then there exists $c \in \mathbb{R}$ such that

$$
|f(n)-c \log n| \leq \varepsilon \quad \text { for all } n \in \mathbb{N} .
$$


In order to express the next result we make some notations. Let $\mathbb{P}$ be the set of all prime numbers and for each $n \in \mathbb{N}$ let $P_{n}=\{p \in \mathbb{P}: p \mid n\}$.

Theorem 3.18. (Kochanek [111]) There is an absolute constant $C \leq \frac{89}{2}$ having the property: if a function $f: \mathbb{N} \rightarrow \mathbb{R}$ satisfies (3.16) and

$$
|f(m)-f(n)| \leq 2 \varepsilon \text { for all } m, n \in \mathbb{N} \text { with } P_{m}=P_{n},
$$

where $\varepsilon \geq 0$ is a fixed constant, then there exists a strongly additive function ${ }^{5}$ $g: \mathbb{N} \rightarrow \mathbb{R}$ such that $|f(n)-g(n)| \leq C \varepsilon$ for all $n \in \mathbb{N}$.

The above theorem gives a stability result for strongly additive functions, but the basic problem remains open.

Problem 3.2. (Kochanek [111]) Assume that $f: \mathbb{N} \rightarrow \mathbb{R}$ satisfies (3.16) with some $\varepsilon \geq 0$. Does there exist an additive arithmetic function $g: \mathbb{N} \rightarrow \mathbb{R}$ such that $|f(n)-g(n)| \leq L \varepsilon$ for all $n \in \mathbb{N}$, where $L$ is an absolute constant?

Some other stability results for additive arithmetic functions one can find in the papers [109-111] by Kochanek.

\subsection{Quadratic functional equation}

In this section we consider the stability problem for the quadratic equation for functions from a space $X$ with an orthogonality relation into a real Banach space. So, our starting point is the conditional functional inequality

$\|f(x+y)+f(x-y)-2 f(x)-2 f(y)\| \leq \varepsilon \quad$ for all $x, y \in X$ with $x \perp y$

or, in more general form,

$\|f(x+y)+f(x-y)-2 f(x)-2 f(y)\| \leq \varphi(x, y) \quad$ for all $x, y \in X$ with $x \perp y$,

for some function $\varphi$ defined on $X^{2}$.

To the best of our knowledge the first result on stability of an orthogonally quadratic functional equation was given by Drljević [62]. His orthogonality relation was defined on a complex Hilbert space $(X,\langle\cdot \mid \cdot\rangle)$ by means of a selfadjoint operator $A: X \rightarrow X$ as follows

$$
x \perp y \quad \text { if and only if }\langle A x \mid y\rangle=0 .
$$

He assumed that the functions involved are continuous and his result states what follows.

\footnotetext{
${ }^{5} \mathrm{~A}$ function $f: \mathbb{N} \rightarrow \mathbb{R}$ is called strongly additive if and only if it satisfies $(2.21)$ and $f\left(p^{n}\right)=$ $f(p)$ for all $n \in \mathbb{N}$ and $p \in \mathbb{P}$.
} 
Theorem 3.19. Let $(X,\langle\cdot \mid \cdot\rangle)$ be a complex Hilbert space, $\operatorname{dim} X \geq 3, A: X \rightarrow X$ a selfadjoint operator, $\operatorname{dim} A(X) \geq 3, \theta \geq 0$ and $p \in[0,2)$ real numbers, and let $f$ be a continuous functional defined on $X$. If

$$
|f(x+y)+f(x-y)-2 f(x)-2 f(y)| \leq \theta\left[|\langle A x \mid x\rangle|^{p / 2}+|\langle A y \mid y\rangle|^{p / 2}\right]
$$

for all $x, y \in X$ with $\langle A x \mid y\rangle=0$ (we assume that $0^{0}=1$ ), then $g: X \rightarrow \mathbb{C}$ well defined by

$$
g(x)=\lim _{n \rightarrow \infty} 2^{-2 n} f\left(2^{n} x\right) \quad \text { for all } x \in X
$$

is a continuous functional such that

$$
g(x+y)+g(x-y)=2 g(x)+2 g(y) \quad \text { for all } x, y \in X \text { with }\langle A x \mid y\rangle=0 .
$$

Furthermore, there exists a real number $\varepsilon>0$ such that

$$
|f(x)-g(x)| \leq|\langle A x \mid x\rangle|^{p / 2} \varepsilon \quad \text { for all } x \in X .
$$

For the case $A=i d$, so with the classical definition of orthogonality defined on an inner product space, and without the continuity assumption of a function mapping now into a Banach space, we have the following (see Sikorska [168, Theorem 5.1]).

Theorem 3.20. Let $(X,\|\cdot\|)$ be a real normed linear space in which the norm comes from an inner product, $\operatorname{dim} X \geq 3$, and let $(Y,\|\cdot\|)$ be a real Banach space. If a function $f: X \rightarrow Y$ satisfies

$$
\|f(x+y)+f(x-y)-2 f(x)-2 f(y)\| \leq \varepsilon\left(\|x\|^{p}+\|y\|^{p}\right)
$$

for all $x, y \in X$ with $x \perp y$, with some $\varepsilon \geq 0$ and $p \in \mathbb{R} \backslash\{2\}$, then there exists a unique quadratic mapping $q: X \rightarrow Y$ such that

$$
\|f(x)-q(x)\| \leq \frac{3 \varepsilon \operatorname{sgn}(p-2)}{2^{p / 2}-2}\|x\|^{p} \quad \text { for all } x \in X_{p},
$$

where $X_{p}=X$ if $p \geq 0$ (with $0^{0}:=1$ ), and $X_{p}=X \backslash\{0\}$ if $p<0$.

The next theorem is based on a result by Moslehian [135] and proves the stability of a pexiderized form of the orthogonally quadratic equation (2.9) for functions defined on an orthogonality space. It does not, however, generalize the previous results since it is assumed that $f$ is odd.

Theorem 3.21. Suppose $(X, \perp)$ is an orthogonality space with a symmetric relation $\perp,(Y,\|\cdot\|)$ is a real Banach space, and $f, g, h: X \rightarrow Y$ are mappings fulfilling

$$
\|f(x+y)+f(x-y)-2 g(x)-2 h(y)\| \leq \varepsilon \quad \text { for all } x, y \in X \text { with } x \perp y,
$$


where $\varepsilon \geq 0$ is a fixed constant. Assume that $f$ is odd. Then there exists exactly one additive mapping $A: X \rightarrow Y$ such that for all $x \in X$,

$$
\begin{aligned}
& \|f(x)-A(x)\| \leq 6 \varepsilon, \\
& \|g(x)-g(0)-A(x)\| \leq 7 \varepsilon, \\
& \|h(x)-h(0)\| \leq \varepsilon .
\end{aligned}
$$

The stability of (2.10) was investigated by Mirzavaziri and Moslehian in [130], also with some additional assumption on $f$.

\subsection{Exponential functional equation}

3.3.1. Formulation of the stability problem. The stability of an (unconditional) exponential equation was first considered by Baker et al. [14] and Baker [13]. They studied a complex valued function $f$ defined on a group, which satisfies the condition

$$
|f(x+y)-f(x) f(y)| \leq \varepsilon
$$

for all $x, y$ and for some fixed $\varepsilon \geq 0$. As a result they obtained that $f$ has to be bounded or exponential.

As observed by Ger [85], taking into account the structure of the equation, it is more appropriate to consider the stability problem starting from the inequality

$$
\left|\frac{f(x+y)}{f(x) f(y)}-1\right| \leq \varepsilon
$$

for a function $f$ with values in $\mathbb{C} \backslash\{0\}$ with some fixed $\varepsilon \geq 0$. In this case there exists an exponential function close in a sense to $f$ (see Ger [85], Ger and Šemrl [88]).

A small modification of this approach is to consider for a complex valued function the system of conditional inequalities

$$
\left|\frac{f(x+y)}{f(x) f(y)}-1\right| \leq \varepsilon \quad \text { for all } x, y \text { with } f(x) f(y) \neq 0
$$

and

$$
\left|\frac{f(x) f(y)}{f(x+y)}-1\right| \leq \varepsilon \quad \text { for all } x, y \text { with } f(x+y) \neq 0
$$

with some nonnegative constant $\varepsilon$ (for this kind of approach in the case of some other functional equation see, e.g., Chudziak [51]).

We were considering this approach while studying the stability of the orthogonally exponential equation (see Sikorska [171]; cf. also Brzdęk and Sikorska [35] and Sikorska [172] for some similar or more general results). 
Theorem 3.22. [171, Corollary 1] Assume that $(X, \perp)$ is an orthogonality space or a real normed space, $\operatorname{dim} X \geq 3$, with James orthogonality. If $f: X \rightarrow \mathbb{K} \in$ $\{\mathbb{R}, \mathbb{C}\}$ satisfies

$$
\left|\frac{f(x+y)}{f(x) f(y)}-1\right| \leq \varepsilon \quad \text { for all } x, y \in X \text { with } x \perp y \text { and } f(x) f(y) \neq 0
$$

and

$$
\left|\frac{f(x) f(y)}{f(x+y)}-1\right| \leq \varepsilon \quad \text { for all } x, y \in X \text { with } x \perp y \text { and } f(x+y) \neq 0
$$

for some nonnegative $\varepsilon<1$, and $f\left(x_{0}\right) \neq 0$ for some $x_{0} \neq 0$, then there exists exactly one $g: X \rightarrow(0, \infty)$ such that

$$
g(x+y)=g(x) g(y) \quad \text { for all } x, y \in X \text { with } x \perp y,
$$

and for all $x \in X$,

$$
\left|\frac{f(x)}{g(x)}-1\right| \leq(1+\varepsilon)^{5}+1 \quad \text { and } \quad\left|\frac{g(x)}{f(x)}-1\right| \leq(1+\varepsilon)^{5}+1 .
$$

Moreover, if $\mathbb{K}=\mathbb{R}$, then for all $x \in X$,

$$
\left|\frac{f(x)}{g(x)}-1\right| \leq(1+\varepsilon)^{5}-1 \quad \text { and } \quad\left|\frac{g(x)}{f(x)}-1\right| \leq(1+\varepsilon)^{5}-1 .
$$

The next result concerns the stability of the pexiderized form of the orthogonally exponential equation.

Theorem 3.23. [171, Corollary 2] Assume that $(X, \perp)$ is an orthogonality space or a real normed space, $\operatorname{dim} X \geq 3$, with the James orthogonality relation. If $f_{1}, f_{2}, f_{3}: X \rightarrow \mathbb{K}$ satisfy

$$
\left|\frac{f_{1}(x+y)}{f_{2}(x) f_{3}(y)}-1\right| \leq \varepsilon \quad \text { for all } x, y \in X \text { with } x \perp y \text { and } f_{2}(x) f_{3}(y) \neq 0,
$$

and

$$
\left|\frac{f_{2}(x) f_{3}(y)}{f_{1}(x+y)}-1\right| \leq \varepsilon \quad \text { for all } x, y \in X \text { with } x \perp y \text { and } f_{1}(x+y) \neq 0 \text {, }
$$

for some nonnegative $\varepsilon<1$, and $f_{1}$ does not vanish on $X \backslash\{0\}$, then there exist functions $g_{1}, g_{2}, g_{3}: X \rightarrow(0, \infty)$ such that

$$
g_{1}(x+y)=g_{2}(x) g_{3}(y) \quad \text { for all } x, y \in X \text { with } x \perp y,
$$

and

$$
\left|\frac{f_{i}(x)}{g_{i}(x)}-1\right| \leq(1+\varepsilon)^{c_{i}}+1 \quad \text { and } \quad\left|\frac{g_{i}(x)}{f_{i}(x)}-1\right| \leq(1+\varepsilon)^{c_{i}}+1
$$

for all $x \in X$ and $i \in\{1,2,3\}$ with $c_{1}=15$ and $c_{2}=c_{3}=16$. 
Moreover, if $\mathbb{K}=\mathbb{R}$, then there exist $\mu_{1}, \mu_{2}, \mu_{3} \in\{-1,1\}$ such that

$$
\left|\frac{\mu_{i} f_{i}(x)}{g_{i}(x)}-1\right| \leq(1+\varepsilon)^{c_{i}}-1 \quad \text { and } \quad\left|\frac{g_{i}(x)}{\mu_{i} f_{i}(x)}-1\right| \leq(1+\varepsilon)^{c_{i}}-1
$$

hold for all $x \in X$ and $i \in\{1,2,3\}$.

3.3.2. Multiplicative arithmetic functions. In the same manner as additive functions we may consider multiplicative arithmetic functions, i.e., functions $f: \mathbb{N} \rightarrow \mathbb{R}$ satisfying the conditional functional equation

$$
f(m n)=f(m) f(n) \quad \text { for all } m, n \in \mathbb{N} \text { with } m \perp_{\mathrm{rp}} n,
$$

and investigate a suitable stability problem.

In the paper by Kochanek and Lewicki [113] the stability problem was formulated by means of a conditional inequality (cf. Baker et al. [14], Baker $[13])$

$$
|f(m n)-f(m) f(n)| \leq \varepsilon \quad \text { for all } m, n \in \mathbb{N} \text { with } m \perp_{\mathrm{rp}} n .
$$

It is clear that every bounded function $f: \mathbb{N} \rightarrow \mathbb{C}$ satisfies (3.21) with some $\varepsilon>0$, so for further investigations we may assume that $f$ is unbounded. Kochanek and Lewicki obtained the following result.

Theorem 3.24. Assume that an unbounded function $f: \mathbb{N} \rightarrow \mathbb{C}$ satisfies (3.21). Then there exists a multiplicative arithmetic function $g: \mathbb{N} \rightarrow \mathbb{C}$ such that

$$
|f(n)-g(n)| \leq \varepsilon \quad \text { for all } n \in \mathbb{N} .
$$

Moreover, if for every $n \in \mathbb{N}$ there is a sequence $\left(n_{k}\right)_{k \in \mathbb{N}} \in \mathbb{N}^{\mathbb{N}}$ such that $n \perp_{\mathrm{rp}} n_{k}$ for $k \in \mathbb{N}$ and $\lim _{k \rightarrow \infty}\left|f\left(n_{k}\right)\right|=\infty$, then $f$ is a multiplicative arithmetic function.

The authors showed also that the estimation in (3.22) is sharp.

As we already know the stability problem may be stated as

$$
\left|\frac{f(m n)}{f(m) f(n)}-1\right| \leq \varepsilon \quad \text { for all } m, n \in \mathbb{N} \text { with } m \perp_{\mathrm{rp}} n,
$$

which is more natural (cf. Ger [85] and Ger and Šemrl [88]).

We cite here a result from Kochanek's paper [111] (one can see some other stability results in Kochanek's [109,110]; for the notations see Sect. 3.1.7 and [111]).

Theorem 3.25. If a function $f: \mathbb{N} \rightarrow \mathbb{C} \backslash\{0\}$ satisfies (3.23) with some $\varepsilon \in[0,1)$ and

$$
\left|\frac{f(m)}{f(n)}-1\right| \leq \rho \quad \text { for all } m, n \in \mathbb{N} \text { with } P_{m}=P_{n}
$$

and with some $\rho \in[0, \sqrt{3} / 2]$ satisfying

$$
\rho \leq \min \left\{2 \varepsilon-\varepsilon^{2}, 2 \varepsilon \sqrt{1-\varepsilon^{2}}\right\},
$$


then there exist a strongly multiplicative function $^{6} g: \mathbb{N} \rightarrow \mathbb{C} \backslash\{0\}$ and a constant $\delta(\varepsilon)$ such that

$$
\left|\frac{f(n)}{g(n)}-1\right| \leq \delta(\varepsilon) \quad \text { and } \quad\left|\frac{g(n)}{f(n)}-1\right| \leq \delta(\varepsilon) \quad \text { for all } n \in \mathbb{N} .
$$

\subsection{Other results}

Obviously, there are many other results concerning stability problems.

Let us just mention one more approach, namely the stability results obtained in non-Archimedean spaces. The field of $p$-adic numbers $\mathbb{Q}_{p}$ is an example of a non-Archimedean space (for details see $[93,160]$ ). It is of great interest for physicists, in particular, in problems coming from quantum physics (see, e.g., [194]).

In [9] (see also [25] and [163, Remark 2] for a more general version), Arriola and Beyer proved the stability of the Cauchy equation for functions from $\mathbb{Q}_{p}$ into $\mathbb{R}$. In [136], Moslehian and Rassias proved the generalized stability of the Cauchy and quadratic functional equations for functions from a semigroup or group, respectively, into a complete non-Archimedean normed space. Najati and Cho [138] were dealing with a suitable problem for Jensen and Pexiderized Cauchy equations.

The stability of functional equations postulated for orthogonal vectors was treated in such spaces by several authors (cf. e.g., Park and Rassias [140]), but since in this survey we restrict ourselves to the classical functional equations, now we will not go into details.

To the best of our knowledge, the stability problems for orthogonal additivity (in its standard form) or orthogonally quadratic equations are open.

\section{Orthogonality equation}

Let $(X,\langle\cdot \mid \cdot\rangle)$ and $(Y,\langle\cdot \mid \cdot\rangle)$ be Hilbert spaces over $\mathbb{K} \in\{\mathbb{R}, \mathbb{C}\}$. A mapping $f: X \rightarrow Y$ is called inner product preserving if $f$ is a solution of the so called orthogonality equation:

$$
\langle f(x) \mid f(y)\rangle=\langle x \mid y\rangle \quad \text { for all } x, y \in X .
$$

One can show (see, e.g., Mlak [133, Lemma 2.1.1 and Remark]) the following.

Theorem 4.1. A function $f$ satisfies the orthogonality equation if and only if it is a linear isometry, i.e., $\|f(x)-f(y)\|=\|x-y\|$ for all $x, y \in X$.

\footnotetext{
${ }^{6}$ A function $f: \mathbb{N} \rightarrow \mathbb{C}$ is called strongly multiplicative if and only if it satisfies (3.20) and $f\left(p^{n}\right)=f(p)$ for all $n \in \mathbb{N}$ and $p \in \mathbb{P}$.
} 
It is easy to see that if a function satisfies the orthogonality equation then it preserves orthogonality, i.e., it satisfies the condition

$$
x \perp y \Rightarrow f(x) \perp f(y) \text { for all } x, y \in X,
$$

where orthogonality is given in a standard way by means of the inner product.

In what follows we present the main results and give some bibliographic data concerning the stability problem. For some more information the reader should consult Chmieliński's survey [42] and the references therein.

\subsection{Stability of the orthogonality equation}

Let $(X,\langle\cdot \mid \cdot\rangle)$ and $(Y,\langle\cdot \mid \cdot\rangle)$ be Hilbert spaces over $\mathbb{K} \in\{\mathbb{R}, \mathbb{C}\}$.

Theorem 4.2. (Chmieliński [42]) If $f: X \rightarrow Y$ satisfies

$$
|\langle f(x) \mid f(y)\rangle-\langle x \mid y\rangle| \leq \varphi(x, y) \quad \text { for all } x, y \in X
$$

with a function $\varphi: X \times X \rightarrow[0, \infty)$ which satisfies the condition

$$
\lim _{m+n \rightarrow \infty} c^{m+n} \varphi\left(c^{-m} x, c^{-n} y\right)=0 \quad \text { for all } x, y \in X
$$

for some $1 \neq c>0$, then there exists a unique mapping $I: X \rightarrow Y$, satisfying the orthogonality equation (4.1) and such that

$$
\|f(x)-I(x)\| \leq \sqrt{\varphi(x, x)} \text { for all } x \in X
$$

This theorem is a generalization of another result obtained by Chmieliński 2 years earlier. Now we can formulate it as a corollary.

Corollary 4.1. (Chmieliński [38]) Let $\varepsilon>0$ and $p \in \mathbb{R} \backslash\{1\}$ be fixed. If a function $f: X \rightarrow Y$ satisfies

$$
|\langle f(x) \mid f(y)\rangle-\langle x \mid y\rangle| \leq \varepsilon\|x\|^{p}\|y\|^{p} \quad \text { for all } x, y \in X_{p},
$$

where $X_{p}=X$ if $p \geq 0$ (with the assumption $0^{0}:=1$ ), and $X_{p}=X \backslash\{0\}$ if $p<0$, then there exists a unique function $I: X \rightarrow Y$ satisfying (4.1) and such that

$$
\|f(x)-I(x)\| \leq \sqrt{\varepsilon}\|x\|^{p} \quad \text { for all } x \in X_{p} .
$$

The next result is a singular case where $p=1$. However, it is proved only for a finite dimensional domain.

Theorem 4.3. (Chmieliński [41]) Let $X$ be finite dimensional. If $f: X \rightarrow Y$ satisfies

$$
|\langle f(x) \mid f(y)\rangle-\langle x \mid y\rangle| \leq \varepsilon\|x\|\|y\| \quad \text { for all } x, y \in X,
$$

then there exists a linear isometry $I: X \rightarrow Y$ such that

$$
\|f(x)-I(x)\| \leq \delta(\varepsilon)\|x\| \quad \text { for all } x \in X
$$

with some continuous mapping $\delta:[0,1) \rightarrow[0, \infty)$ satisfying $\lim _{\varepsilon \rightarrow 0^{+}} \delta(\varepsilon)=0$. 
Problem 4.1. Does the assertion of Theorem 4.3 remain valid in the case $X$ is infinite dimensional?

Remark 4.1. (a) If $X=Y=\mathbb{R}^{n}$ and $p \in \mathbb{R} \backslash\{1\}$, then $f=I$ (hyperstability phenomenon) [38]. (b) If $X=Y=\mathbb{R}^{n}$ and $p=1$, then we have stability, but there is no hyperstability effect [41].

The stability/hyperstability effect concerning the dimension can be explained via results of Badora and Chmieliński [11] about the decomposition of solutions of (4.3).

Theorem 4.4. Let $(X,\langle\cdot \mid \cdot\rangle)$ and $(Y,\langle\cdot \mid \cdot\rangle)$ be Hilbert spaces and let $f: X \rightarrow Y$ satisfy (4.3) with $\varphi$ satisfying (4.4). Then there exists a closed linear subspace $H$ of $Y$ such that

$$
\begin{aligned}
& f(x)=P_{H} f(x)+P_{H^{\perp}} f(x) \quad \text { for all } x \in X, \\
& \left\|P_{H^{\perp}} f(x)\right\| \leq \sqrt{\varphi(x, x)} \quad \text { for all } x \in X
\end{aligned}
$$

and

$$
\left\langle P_{H} f(x) \mid P_{H} f(y)\right\rangle=\langle x \mid y\rangle \text { for all } x, y \in X,
$$

where $P_{H}, P_{H^{\perp}}$ denote the orthogonal projections from $Y$ onto $H$ and $H^{\perp}$, respectively.

Conversely, if $f: X \rightarrow Y$ is given and there exists a closed linear subspace $H$ of $Y$ such that the above three conditions hold, then

$$
|\langle f(x) \mid f(y)\rangle-\langle x \mid y\rangle| \leq\left\|P_{H^{\perp}} f(x)\right\|\left\|P_{H^{\perp}} f(y)\right\| \quad \text { for all } x, y \in X .
$$

The stability result is included in the above theorem. On the other hand, since the subspace $H$ is isomorphic to $X$, in the case $\operatorname{dim} X=\operatorname{dim} Y<\infty$, we get $H=Y$ and hence, the hyperstability.

A similar stability problem can be also stated in Hilbert modules (cf. Chmieliński and Moslehian [46]).

\subsection{Orthogonality equation almost everywhere}

For the orthogonality equation (4.1) we may study its "almost everywhere" version (in the sense of ideals). Namely, we consider

$$
\langle f(x) \mid f(y)\rangle=\langle x \mid y\rangle \quad \text { for all }(x, y) \in X^{2} \backslash M
$$

or

$$
\langle f(x) \mid f(y)\rangle=\langle x \mid y\rangle \text { for all } x, y \in X \backslash U,
$$

where $M$ and $U$ are in some sense small in $X^{2}$ and $X$, respectively. 
In the papers by Chmieliński and Rätz [47] and Chmieliński and Ger [45], the authors showed (sometimes under some additional assumptions) the existence of a unique solution of the orthogonality equation which is equal to $f$ almost everywhere on $X$.

In [37], Chmieliński proved the stability of the orthogonality equation postulated almost everywhere.

\section{Preserving orthogonality}

\subsection{Exact orthogonality preserving mappings}

As it was already mentioned, we say that $f: X \rightarrow Y$ (exactly) preserves orthogonality if and only if it satisfies (4.2).

Remark 5.1. Such mappings can be very irregular, far from being continuous or linear. For that reason we restrict ourselves to linear mappings only.

We start our considerations with inner product spaces and with the standard orthogonality defined by means of the inner product.

Theorem 5.1. (Chmieliński [39]) Let $(X,\langle\cdot \mid \cdot\rangle),(Y,\langle\cdot \mid \cdot\rangle)$ be (real or complex) inner product spaces. For a nonzero linear mapping $f: X \rightarrow Y$ the following conditions are equivalent with some $\gamma>0$ :

(i) $f$ preserves orthogonality;

(ii) $\|f(x)\|=\gamma\|x\|$ for all $x \in X$;

(iii) $\langle f(x) \mid f(y)\rangle=\gamma^{2}\langle x \mid y\rangle$ for all $x, y \in X$.

We continue with presenting results for normed spaces. For the orthogonality relation in the sense of Birkhoff the first result comes from Koldobsky [117]. He showed that also in the case of a real normed space $(X,\|\cdot\|)$ a linear mapping $f: X \rightarrow X$ preserving orthogonality has to be a similarity, i.e., there exists a positive constant $\gamma$ such that

$$
\|f(x)\|=\gamma\|x\| \text { for all } x \in X .
$$

Clearly, for a linear mapping $f$, we have then $\gamma=\|f\|$.

The respective result for both real and complex cases was given by Blanco and Turnšek in [27].

Theorem 5.2. Let $(X,\|\cdot\|)$ and $(Y,\|\cdot\|)$ be (real or complex) normed linear spaces and let $f: X \rightarrow Y$ be a linear operator. Then $f$ preserves the Birkhoff orthogonality if and only if $f$ is a similarity.

Six years later, using the connections between the Birkhoff orthogonality and norm derivatives, Ionică [101] gave an alternative proof of the above results in the case of different real normed spaces.

The result concerning James orthogonality is almost immediate. 
Theorem 5.3. (Chmieliński and Wójcik [48]) Let $(X,\|\cdot\|)$ and $(Y,\|\cdot\|)$ be normed linear spaces and let $f: X \rightarrow Y$ be a linear operator. The following conditions are equivalent:

(a) there exists $\gamma>0$ such that $\|f(x)\|=\gamma\|x\|$ for all $x \in X$;

(b) $x \perp_{J} y \Rightarrow f(x) \perp_{J} f(y)$ for all $x, y \in X$;

(c) $x \perp_{J} y \Leftrightarrow f(x) \perp_{J} f(y)$ for all $x, y \in X$.

Koehler and Rosenthal [116] have shown that a linear operator from a normed space into itself is an isometry if and only if it preserves some semiinner product. We present here a slightly more general result.

Theorem 5.4. (Chmieliński [43]) Let $(X,\|\cdot\|)$ and $(Y,\|\cdot\|)$ be normed linear spaces and let $f: X \rightarrow Y$ be a linear operator. Then $f$ is a similarity (i.e., (5.1) is satisfied with some $\gamma>0$ ) if and only if there exist semi-inner products $[\cdot \cdot \cdot]_{X}$ and $[\cdot \mid \cdot]_{Y}$ in $X$ and $Y$, respectively, such that

$$
[f(x) \mid f(y)]_{Y}=\gamma^{2}[x \mid y]_{X} \quad \text { for all } x, y \in X .
$$

Moreover, if $X=Y$ (with the same norm), then the assertion holds with the same semi-inner product.

The next theorem gives results concerning the case of $\rho_{+^{-}}, \rho_{-}$and $\rho_{-}$ orthogonalities (cf., Chmieliński and Wójcik [49, Theorem 5] and Wójcik [195, Theorem 4.3]).

Theorem 5.5. Let $(X,\|\cdot\|)$ and $(Y,\|\cdot\|)$ be normed linear spaces and let $f: X \rightarrow$ $Y$ be a nonzero linear operator. Then the following conditions are equivalent with some $\gamma>0$ :

(a) $f$ preserves $\rho_{+}$-orthogonality;

(b) $f$ preserves $\rho_{-}$-orthogonality;

(c) $f$ preserves $\rho$-orthogonality;

(d) $\|f(x)\|=\gamma\|x\|$ for all $x \in X$;

(e) $\rho_{+}^{\prime}(f(x), f(y))=\gamma^{2} \rho_{+}^{\prime}(x, y)$ for all $x, y \in X$;

(f) $\rho_{-}^{\prime}(f(x), f(y))=\gamma^{2} \rho_{-}^{\prime}(x, y)$ for all $x, y \in X$;

(g) $\rho^{\prime}(f(x), f(y))=\gamma^{2} \rho^{\prime}(x, y)$ for all $x, y \in X$.

Ilišević and Turnšek [100] studied orthogonality preserving mappings in the setting of inner product $C^{*}$-modules. In particular, if $X$ and $Y$ are inner product $C^{*}$-modules over the $C^{*}$-algebra $\mathcal{A}$, any scalar multiple of an $\mathcal{A}$-linear isometry is an $\mathcal{A}$-linear orthogonality preserving mapping. In particular, we have [100, Proposition 2.3].

Theorem 5.6. Let $\mathcal{A}$ be a $C^{*}$-algebra and let $(X,\langle\cdot \mid \cdot\rangle),(Y,\langle\cdot \mid \cdot\rangle)$ be inner product $\mathcal{A}$-modules. For a mapping $f: X \rightarrow Y$ and some $\gamma>0$ the following assertions are equivalent:

(i) $f$ is $\mathcal{A}$-linear and $\|f(x)\|=\gamma\|x\|$ for all $x \in X$;

(ii) $\langle f(x) \mid f(y)\rangle=\gamma^{2}\langle x \mid y\rangle$ for all $x, y \in X$. 
Furthermore, each of these assertions implies:

(iii) $f$ is $\mathcal{A}$-linear and orthogonality preserving.

If $X=Y$ then (i) and (ii) are also equivalent to

(iv) $f$ is $\mathcal{A}$-linear and there exists a semi-inner product $[\cdot \mid \cdot]: X \times X \rightarrow \mathbb{C}$ satisfying $[f(x) \mid f(y)]=\gamma^{2}[x \mid y]$ for all $x, y \in X$.

As it is shown in [100, Example 2.4], the converse does not hold in general, that is (iii) is not equivalent to (i) and (ii) in general. It holds, however, if $\mathcal{A}$ contains $\mathcal{K}(\mathcal{H})$ (the $C^{*}$-algebra of all compact operators on a Hilbert space $\mathcal{H}$ ) (see [100, Theorem 3.1]).

\subsection{Mappings which approximately preserve orthogonality}

Let $\varepsilon \in[0,1)$. We say that $f: X \rightarrow Y \varepsilon$-approximately preserves orthogonality if and only if

$$
x \perp y \Rightarrow f(x) \perp^{\varepsilon} f(y) \quad \text { for all } x, y \in X,
$$

and we say that $f$ approximately preserves orthogonality if it $\varepsilon$-approximately preserves orthogonality with some $\varepsilon \in[0,1)$.

Further, we say that a linear mapping $f: X \rightarrow Y$ between normed spaces is an approximate isometry if there exists $\varepsilon>0$ (we speak then of an $\varepsilon$-isometry) such that $|\|f(x)\|-\|x\|| \leq \varepsilon\|x\|$ for all $x \in X$. A more general definition is given by Mojškerc and Turnšek [134]: $f: X \rightarrow Y$ is a general approximate isometry if and only if there exist mappings $\delta_{1}, \delta_{2}:[0, \infty) \rightarrow[0, \infty)$ with the property $\delta_{1}(\varepsilon), \delta_{2}(\varepsilon) \rightarrow 0$ as $\varepsilon \rightarrow 0$ and such that

$$
\left(1-\delta_{1}(\varepsilon)\right)\|x\| \leq\|f(x)\| \leq\left(1+\delta_{2}(\varepsilon)\right)\|x\| \text { for all } x \in X .
$$

Finally, we say that $f$ is a (general) approximate similarity if it is a scalar multiple of a (general) approximate isometry. More exactly, considering the known approximations, we say that a linear and continuous mapping $f$ is an $\eta$-similarity if

$$
(1-\eta)\|f\|\|x\| \leq\|f(x)\| \leq\|f\|\|x\| \text { for all } x \in X .
$$

As usual, first we present results for inner product spaces (Chmieliński [39, Theorem 2]; see also Turnšek [189, Remark 2.1]).

Theorem 5.7. Let $(X,\langle\cdot \mid \cdot\rangle),(Y,\langle\cdot \mid \cdot\rangle)$ be inner product spaces and $f: X \rightarrow Y$ be a linear mapping satisfying (5.2) with some $\varepsilon \in[0,1)$. Then $f$ is injective, continuous and, with some $\gamma>0, f$ satisfies the inequality

$|\langle f(x) \mid f(y)\rangle-\gamma\langle x \mid y\rangle| \leq \frac{4 \varepsilon}{1+\varepsilon} \min \{\gamma\|x\|\|y\|,\|f(x)\|\|f(y)\|\}$ for all $x, y \in X$.

Conversely, if $f: X \rightarrow Y$ satisfies

$$
|\langle f(x) \mid f(y)\rangle-\gamma\langle x \mid y\rangle| \leq \varepsilon \min \{\gamma\|x\|\|y\|,\|f(x)\|\|f(y)\|\} \quad \text { for all } x, y \in X
$$


with some $\varepsilon>0$ and with some $\gamma>0$, then $f$ is a quasi-linear mapping ${ }^{7}$, which approximately preserves orthogonality.

As a corollary we derive the fact that each linear mapping which is an $\varepsilon$-approximately preserving orthogonality, is a continuous $\left(1-\sqrt{\frac{1-\varepsilon}{1+\varepsilon}}\right)$-similarity (see Chmieliński [39], Turnšek [189]). The converse is also true: each continuous $\varepsilon$-similarity is a mapping that $\varepsilon$-approximately preserves orthogonality (Wójcik [196,197]).

Kong and Cao [119] were studying a more general case, namely $(\delta, \varepsilon)$-approximately orthogonality preserving linear mappings, i.e., mappings satisfying

$$
x \perp^{\delta} y \Rightarrow f(x) \perp^{\varepsilon} f(y) \quad \text { for all } x, y \in X
$$

with some $\delta, \varepsilon \in[0,1)$. They proved that such mappings are bounded. Moreover, they gave a sufficient condition for a linear mapping to be $(\delta, \varepsilon)$-approximately orthogonality preserving. We cite here two theorems from [119].

Theorem 5.8. Let $(X,\langle\cdot \mid \cdot\rangle),(Y,\langle\cdot \mid \cdot\rangle)$ be Hilbert spaces and $g: X \rightarrow Y$ be a linear orthogonality preserving mapping. If there is a linear mapping $f: X \rightarrow$ $Y$ such that

$$
\|f(x)-g(x)\| \leq \eta\|g(x)\| \text { for all } x \in X,
$$

with some $\eta \in\left[0, \frac{1}{4}\right)$, then for every $\delta \in[0,1-4 \eta)$, the function $f$ is $(\delta, \varepsilon)$ approximately orthogonality preserving, where $\varepsilon=\frac{\eta(\eta+2)+\delta}{(1-\eta)^{2}}$.

Theorem 5.9. Let $(X,\langle\cdot \mid \cdot\rangle),(Y,\langle\cdot \mid \cdot\rangle)$ be Hilbert spaces, $\delta, \varepsilon \in[0,1)$ and let $f: X \rightarrow Y$ be a linear $(\delta, \varepsilon)$-approximately orthogonality preserving mapping. Then $f$ is continuous and there exists $\lambda_{0} \in \mathbb{C}$ such that

$$
\mu\|f\|\|x\| \leq\|f(x)\| \leq\|f\|\|x\| \quad \text { for all } x \in X,
$$

where $\mu=\frac{\left|\lambda_{0}\right|}{\delta+1} \sqrt{\frac{1-\varepsilon}{1+\varepsilon}}>0$.

Wójcik showed (see $[196,197]$ ) that if $f$ is a linear $(\delta, \varepsilon)$-approximately orthogonality preserving mapping between two complex Hilbert spaces, then $\delta \leq \varepsilon$ (which is not derivable from [119]), $f$ is continuous, injective and satisfies (5.4) with $\mu=\sqrt{\frac{1-\varepsilon}{1+\varepsilon}} \sqrt{\frac{1+\delta}{1-\delta}}$.

The following results already concern normed linear spaces. We start with results concerning James orthogonality.

\footnotetext{
${ }^{7} \mathrm{~A}$ function $f$ is called quasi-linear if it satisfies with some $\delta, \eta \geq 0$ the conditions $\| f(x+$ $y)-f(x)-f(y) \| \leq \delta(\|x\|+\|y\|)$ and $\|f(\lambda x)-\lambda f(x)\| \leq \eta|\lambda|\|x\|$ for all $x, y \in X$ and $\lambda \in \mathbb{K}$.
} 
Theorem 5.10. (Chmieliński and Wójcik [48, Theorem 3.2]) Let $(X,\|\cdot\|)$, $(Y,\|\cdot\|)$ be real normed linear spaces, $\varepsilon \in[0,1)$ and let $f: X \rightarrow Y$ be a nontrivial linear mapping satisfying

$$
x \perp_{J} y \Rightarrow f(x)^{\varepsilon} \perp_{J} f(y) \quad \text { for all } x, y \in X .
$$

Then $f$ is injective, continuous and satisfies

$$
\frac{1-\varepsilon}{1+\varepsilon}\|f\|\|x\| \leq\|f(x)\| \leq \frac{1+\varepsilon}{1-\varepsilon}[f]\|x\| \quad \text { for all } x \in X,
$$

where $[f]=\inf \{\|f(x)\|:\|x\|=1\}=\sup \{M \geq 0:\|f(x)\| \geq M\|x\|, x \in X\}$, or equivalently,

$$
\frac{1-\varepsilon}{1+\varepsilon} \gamma\|x\| \leq\|f(x)\| \leq \frac{1+\varepsilon}{1-\varepsilon} \gamma\|x\| \quad \text { for all } x \in X, \gamma \in[[f],\|f\|] .
$$

Conversely, if a linear bounded mapping $f: X \rightarrow Y$ satisfies (5.6) (or (5.7)), then it satisfies (5.5).

Obviously, (5.6) holds for a linear mapping $f: X \rightarrow Y$ satisfying a stronger condition than (5.5), i.e., the condition $x \perp_{J} y \Rightarrow f(x) \perp_{J}^{\varepsilon} f(y)$ for all $x, y \in X$.

As a corollary, we derive that linear approximately James orthogonality preserving mappings are approximate similarities. And also each $\varepsilon$-isometry $\varepsilon$-approximately preserves James orthogonality with respect to the relation $\varepsilon_{J}$.

Problem 5.1. Is it true that each $\varepsilon$-isometry (or more generally, $\varepsilon$-similarity) $\varepsilon$-approximately preserves James orthogonality with respect to the relation $\perp \stackrel{\varepsilon}{J} ?$

Some further properties of functions satisfying (5.5) are collected in Chmieliński and Wójcik [48, Theorem 3.6].

Mojškerc and Turnšek [134] were dealing with mappings approximately preserving Birkhoff orthogonality with respect to the relation $\perp_{B}^{\varepsilon}$. They showed that such mappings have to be approximate similarities. Namely, they proved the following.

Theorem 5.11. Let $(X,\|\cdot\|),(Y,\|\cdot\|)$ be normed linear spaces, $\varepsilon \in\left[0, \frac{1}{16}\right)$ and let $f: X \rightarrow Y$ be a linear mapping satisfying

$$
x \perp_{B} y \Rightarrow f(x) \perp_{B}^{\varepsilon} f(y) \text { for all } x, y \in X .
$$

Then $f$ is continuous and

$$
(1-16 \varepsilon)\|f\|\|x\| \leq\|f(x)\| \leq\|f\|\|x\| \quad \text { for all } x \in X .
$$

In the case of real spaces the constant $(1-16 \varepsilon)$ in the above theorem can be replaced by $(1-8 \varepsilon)$ with $\varepsilon \in\left[0, \frac{1}{8}\right)$. 
However, in contrast to inner product spaces or normed spaces with James orthogonality, the converse theorem is not true, which was proved by Wójcik [196] (see also Mojškerc and Turnšek [134]).

Surprisingly, the situation looks different in the case of the relation ${ }^{\varepsilon} \perp_{B}$ defined by Dragomir in [58]. Here we know that an approximate similarity is an approximately Birkhoff orthogonality preserving mapping.

Theorem 5.12. (Mojškerc and Turnšek [134, Proposition 3.6]) Let $(X,\|\cdot\|)$, $(Y,\|\cdot\|)$ be normed linear spaces and $f: X \rightarrow Y$ be a scalar multiple of a general linear approximate isometry. Then

$$
x \perp_{B} y \Rightarrow f(x)^{\delta(\varepsilon)} \perp_{B} f(y) \text { for all } x, y \in X,
$$

where $\delta(\varepsilon)=\frac{\delta_{1}(\varepsilon)+\delta_{2}(\varepsilon)}{1+\delta_{2}(\varepsilon)}$.

However, we do not know the complete answer for the question about the converse. It is known only that an approximately Birkhoff orthogonality preserving mapping is bounded (Mojškerc and Turnšek [134]). Moreover, if $X$ and $Y$ are normed spaces and $Y$ is uniformly smooth, then a linear mapping satisfying

$$
x \perp_{B} y \Rightarrow f(x)^{\varepsilon} \perp_{B} f(y) \text { for all } x, y \in X
$$

is a scalar multiple of an approximate isometry ([134]).

In his doctorial dissertation [196], Wójcik obtained the same assertion for some other examples of spaces. The general situation remains then open, so we may state the next

Problem 5.2. Are approximately Birkhoff orthogonality preserving mappings in the sense of relation ${ }^{\varepsilon} \perp_{B}$ approximate similarities?

Chmieliński and Wójcik [50] studied approximately $\rho$-orthogonality preserving mappings. They proved a property that for arbitrary normed spaces $X$ and $Y$, if a linear mapping $f: X \rightarrow Y$ approximately preserves $\rho_{+}$-orthogonality or approximately preserves $\rho_{-}$-orthogonality then it approximately preserves Birkhoff orthogonality with respect to the relation $\perp_{B}^{\varepsilon}$. Later, Wójcik [196] showed that, in fact, all four properties: approximate $\rho_{+}\left(\rho_{-}, \rho\right.$, Birkhoff $)-$ orthogonality preservation, are equivalent. These results, together with the result of Mojškerc and Turnšek concerning Birkhoff orthogonality give

Theorem 5.13. (Chmieliński and Wójcik [50]; Wójcik [196]) Let $(X,\|\cdot\|)$, $(Y,\|\cdot\|)$ be real normed linear spaces, $\varepsilon \in\left[0, \frac{1}{8}\right)$ and let $f: X \rightarrow Y$ be a linear mapping satisfying one of the following conditions:

$$
\begin{aligned}
& x \perp_{\rho_{+}} y \Rightarrow f(x) \perp_{\rho_{+}}^{\varepsilon} f(y) \quad \text { for all } x, y \in X, \\
& x \perp_{\rho_{-}} y \Rightarrow f(x) \perp_{\rho_{-}}^{\varepsilon} f(y) \text { for all } x, y \in X, \\
& x \perp_{\rho} y \Rightarrow f(x) \perp_{\rho}^{\varepsilon} f(y) \quad \text { for all } x, y \in X .
\end{aligned}
$$


Then

$$
(1-8 \varepsilon)\|f\|\|x\| \leq\|f(x)\| \leq\|f\|\|x\| \quad \text { for all } x \in X .
$$

We proceed to the investigations concerning Roberts orthogonality. Suppose that $(X,\|\cdot\|)$ and $(Y,\|\cdot\|)$ are real normed spaces. Analogously as before, we will say that a linear mapping $f: X \rightarrow Y(\delta, \eta)$-approximately preserves Roberts orthogonality if and only if

$$
x^{\delta} \perp_{R} y \Rightarrow f(x)^{\eta} \perp_{R} f(y) \text { for all } x, y \in X .
$$

Zamani and Moslehian in [201] gave several sufficient conditions for a linear mapping to be $(\delta, \eta)$-approximately preserving Roberts orthogonality. We cite here one of the results.

Theorem 5.14. (Zamani and Moslehian [201, Theorem 3.1]) Let $f: X \rightarrow Y$ be a scalar multiple of a general linear approximate isometry, i.e., $f=\lambda U$ and $\left(1-\varphi_{1}(\varepsilon)\right)\|x\| \leq\|U x\| \leq\left(1+\varphi_{2}(\varepsilon)\right)\|x\|$, for all $x \in X$, where $\varphi_{1}(\varepsilon) \rightarrow 0$ and $\varphi_{2}(\varepsilon) \rightarrow 0$ as $\varepsilon \rightarrow 0$. Then $f$ is a $(\delta, \eta)$-approximately Roberts orthogonality preserving mapping for any $\delta$ and with $\eta=\frac{2 \delta+(1-\delta) \varphi_{1}(\varepsilon)+(1+\delta) \varphi_{2}(\varepsilon)}{2-(1-\delta) \varphi_{1}(\varepsilon)+(1+\delta) \varphi_{2}(\varepsilon)}$.

So, it means that a scalar multiple of a general linear approximate isometry is a $(\delta, \varepsilon)$-approximately Roberts orthogonality preserving mapping.

The case of $C^{*}$-modules was carried out by Ilišević and Turnšek [100]. Namely, they proved the following [100, Theorem 3.7].

Theorem 5.15. Let $\mathcal{A}$ be a $C^{*}$-algebra such that $\mathcal{K}(\mathcal{H}) \subseteq \mathcal{A} \subseteq \mathcal{B}(\mathcal{H})$, where $\mathcal{K}(\mathcal{H}), \mathcal{B}(\mathcal{H})$ denote the $C^{*}$-algebra of all compact operators and the $C^{*}$-algebra of all bounded operators on a Hilbert space $\mathcal{H}$, respectively, and let $X, Y$ be inner product $\mathcal{A}$-modules. Let $f: X \rightarrow Y$ be an $\mathcal{A}$-linear mapping satisfying with some $\varepsilon \in[0,1)$ the condition

$$
\langle x \mid y\rangle=0 \Rightarrow\|\langle f(x) \mid f(y)\rangle\| \leq \varepsilon\|f(x)\|\|f(y)\| \quad \text { for all } \quad x, y \in X .
$$

Then $f$ is bounded and

$$
\|\langle f(x) \mid f(y)\rangle-\| f\left\|^{2}\langle x \mid y\rangle\right\| \leq \frac{4 \varepsilon}{1+\varepsilon}\|f\|^{2}\|x\|\|y\| \quad \text { for all } x, y \in X .
$$

\subsection{Stability of the orthogonality preserving property}

Coming to a stability problem again, now we answer the question: given a linear approximately orthogonality preserving mapping $f$, does there exist a linear orthogonality preserving mapping close (in a sense) to $f$ ? From the last two sections we already know that in the considered cases such mappings are continuous.

The first (positive) answer was given in the case of finite dimensional inner product spaces by Chmieliński [41]. Using another approach in the proof, based 
on operator theory in Hilbert spaces, Turnšek got rid of that assumption and obtained the following result.

Theorem 5.16. (Chmieliński [41], Turnšek [189]) Let $(X,\langle\cdot \mid \cdot\rangle),(Y,\langle\cdot \mid \cdot\rangle)$ be Hilbert spaces. For a linear, $\varepsilon$-approximately orthogonality preserving mapping $f: X \rightarrow Y$ there exists a linear orthogonality preserving operator $g: X \rightarrow Y$ such that

$$
\|f-g\| \leq\left(1-\sqrt{\frac{1-\varepsilon}{1+\varepsilon}}\right) \min \{\|f\|,\|g\|\} .
$$

Problem 5.3. Is the completeness of $X$ necessary? That is, is the above result true for non-complete spaces?

Considering now Hilbert modules, we have the following result (for the notation see Theorerm 5.15).

Theorem 5.17. (Ilišević and Turnšek [100, Theorem 4.4]) Let $\mathcal{A}=\mathcal{K}(\mathcal{H})$ and let $X, Y$ be Hilbert $\mathcal{A}$-modules. If $f: X \rightarrow Y$ is an $\mathcal{A}$-linear, $\varepsilon$-approximately orthogonality preserving mapping with some $\varepsilon \in[0,1)$, then there exists an $\mathcal{A}$-linear isometry $I: X \rightarrow Y$ such that

$$
\|f-\| f\|I\| \leq\left(1-\sqrt{\frac{1-\varepsilon}{1+\varepsilon}}\right)\|f\| .
$$

Problem 5.4. (Ilišević and Turnšek [100]) Is the above result valid for an arbitrary $C^{*}$-algebra $\mathcal{A}$ such that $\mathcal{K}(\mathcal{H}) \subset \mathcal{A} \subset \mathcal{B}(\mathcal{H})$ ?

Before presenting results concerning the stability of the orthogonality preserving property in normed spaces, we give some necessary facts on the stability of isometries. That problem is formulated as follows:

For a pair $(X, Y)$ of normed spaces, does there exist a function $\delta:[0,1) \rightarrow$ $[0, \infty)$ satisfying $\lim _{\varepsilon \rightarrow 0} \delta(\varepsilon)=0$ and such that for any linear $\varepsilon$-isometry $f: X \rightarrow Y$ (with $\varepsilon \in[0,1)$ ) there exists a linear isometry $I: X \rightarrow Y$ such that $\|f-I\| \leq \delta(\varepsilon)$ ?

Denote by $\mathfrak{A}$ the class of all pairs of normed spaces for which the above problem has an affirmative answer (for some details see, e.g., Ding [57] or Protasov [145]).

Let $(X,\|\cdot\|),(Y,\|\cdot\|)$ be (real or complex) normed spaces, $f: X \rightarrow Y$ be linear and $\varepsilon \in[0,1)$. We will formulate the stability results for the property of orthogonality preservation for various notions of orthogonality.

Theorem 5.18. (Chmieliński, Wójcik [48, Theorem 5.2]) Let $(X, Y) \in \mathfrak{A}$ with a suitable mapping $\delta:[0,1) \rightarrow[0, \infty)$ satisfying $\lim _{\varepsilon \rightarrow 0} \delta(\varepsilon)=0$. Let $f: X \rightarrow$ $Y$ be a linear mapping satisfying (5.5). Then there exists a linear mapping $g: X \rightarrow Y$ preserving James orthogonality such that

$$
\|f-g\| \leq \delta(\varepsilon) \min \{\|f\|,\|g\|\} .
$$


In fact, also the converse can be proved: if for real normed spaces $X$ and $Y$, the James orthogonality preserving property is stable, then $(X, Y) \in \mathfrak{A}$ (see Chmieliński [44], Wójcik [196]). So, the two properties are equivalent.

A suitable example of spaces for which the stability of the above property cannot be proved is given by Chmieliński [44] (see also Protasov [145]).

From Theorem 5.18, it follows that the property of orthogonality preservation is also stable if we consider the assumption with respect to the relation $\perp \stackrel{\varepsilon}{J}$.

In the case of Birkhoff orthogonality an answer to the stability problem was given by Mojškerc and Turnšek [134, Theorem 4.1].

Theorem 5.19. Assume that $(X, Y) \in \mathfrak{A}$ and let $f: X \rightarrow Y$ be a linear mapping satisfying (5.8). Then there exists a linear mapping $g: X \rightarrow Y$ preserving the Birkhoff orthogonality and such that

$$
\|f-g\| \leq \delta(\varepsilon)\|f\|
$$

with some function $\delta$ (depending only on $X$ and $Y$ ) satisfying $\lim _{\varepsilon \rightarrow 0^{+}} \delta(\varepsilon)=$ 0 .

Problem 5.5. Is the converse true, that is, for (real) normed spaces $X$ and $Y$, if the Birkhoff orthogonality preserving property is stable (with approximation given by the relation $\left.\perp_{B}^{\varepsilon}\right)$, does it necessarily follow that $(X, Y) \in \mathfrak{A}$ ?

In the case of the approximation given by the relation $\perp_{B}$, if $X$ and $Y$ are normed spaces such that the stability of the orthogonality preserving property holds, then $(X, Y) \in \mathfrak{A}$ (Mojškerc and Turnšek [134, Proposition 4.2]).

In case $Y$ is uniformly smooth, the two kinds of stability properties and the property $(X, Y) \in \mathfrak{A}$ are equivalent (Mojškerc and Turnšek [134, Theorem 4.3]).

If $X$ and $Y$ are finite dimensional normed spaces and $f: X \rightarrow Y$ approximately preserves orthogonality in the sense of ${ }^{\varepsilon} \perp_{B}$, then it is close to a multiple of a linear isometry, i.e., it satisfies (5.9) (Mojškerc and Turnšek [134, Proposition 4.4]).

In [196], Wójcik gave some other conditions imposed on the spaces $X$ and $Y$ which imply that the Birkhoff orthogonality preserving property is stable (with approximation given by the relation ${ }^{\varepsilon} \perp_{B}$ ) as well as he showed an example of spaces for which the Birkhoff orthogonality preserving property is not stable.

Problem 5.6. Describe the set of all pairs $(X, Y)$, for which the Birkhoff orthogonality preserving property (in the sense of ${ }^{\varepsilon} \perp_{B}$ ) is stable.

Similarly as above, the stability problem of the property of preserving the $\rho$ $\left(\rho_{+}, \rho_{-}\right)$-orthogonality is connected with the property of approximate orthogonality preservation as well as with the stability of isometries for given spaces. 
Theorem 5.20. (Wójcik [196]) Assume that $(X,\|\cdot\|)$ and $(Y,\|\cdot\|)$ are normed spaces such that $(X, Y) \in \mathfrak{A}$ and let $f: X \rightarrow Y$ be a linear mapping which approximately preserves $\rho$-orthogonality, that is, satisfies the condition

$$
x \perp_{\rho} y \Rightarrow f(x) \perp_{\rho}^{\varepsilon} f(y) \quad \text { for all } x, y \in X .
$$

Then there exists a linear mapping $g: X \rightarrow Y$ preserving $\rho$-orthogonality and such that (5.9) holds with some function $\delta$ (depending only on $X$ and $Y$ ) satisfying $\lim _{\varepsilon \rightarrow 0^{+}} \delta(\varepsilon)=0$.

Since, by Theorem 5.5, the properties of preserving $\rho_{-}, \rho_{+^{-}}$and $\rho_{--}$ orthogonality are equivalent as well as the corresponding properties of approximate preservations, the above theorem can be stated in the same form also for $\rho_{+-}$and $\rho_{-}$-orthogonality.

\section{Acknowledgements}

The topic of the article is by no means completed. It is impossible to touch upon all problems connected with orthogonalities and functional equations in a single manuscript, so just a selection of them was given. As it was already mentioned at the very beginning, we were presenting results concerning merely classical functional equations.

The starting point for writing this article was a survey talk given during the 51st International Symposium on Functional Equations held in Rzeszów, Poland, in June 2013. The author is grateful to the Scientific Committee of the Symposium chaired by Professor Roman Ger for the invitation to give this talk. The author would also like to thank Professor Karol Baron for helpful hints, comments and fruitful discussion while preparing the final version of this manuscript. Last, but not least, many thanks to the referee for several helpful remarks.

Open Access This article is distributed under the terms of the Creative Commons Attribution License which permits any use, distribution, and reproduction in any medium, provided the original author(s) and the source are credited.

\section{References}

[1] Aczél, J., Dhombres, J.: Functional Equations in Several Variables. Encyclopedia Math. Appl., vol. 31. Cambridge University Press, Cambridge (1989)

[2] Alonso, J., Benítez, C.: Orthogonality in normed linear spaces: a survey. Part I: main properties. Extracta Math. 3, 1-15 (1988)

[3] Alonso, J., Benítez, C.: Orthogonality in normed linear spaces: a survey. Part II: relations between main orthogonalities. Extracta Math. 4, 121-131 (1989)

[4] Alonso, J., Martini, H., Wu, S.: On Birkhoff orthogonality and isosceles orthogonality in normed linear spaces. Aequationes Math. 83, 153-189 (2012) 
[5] Alsina, C., Cruells, P., Tomás, M.S.: Isosceles trapezoids, norms and inner products. Arch. Math. (Basel) 72, 233-240 (1999)

[6] Alsina, C., Sikorska, J., Tomás, M.S.: On the equation of the $\rho$-orthogonal additivity. Comment. Math. Prace Mat. 47(2), 171-178 (2007)

[7] Alsina, C., Sikorska, J., Tomás, M.S.: Norm Derivatives and Characterizations of Inner Product Spaces. World Scientific Publishing Co., New Jersey (2010)

[8] Amir, D.: Characterizations of Inner Product Spaces. In: Operator Theory: Advances and Applications, vol. 20. Birkhäuser, Basel (1986)

[9] Arriola, L.M., Beyer, W.A.: Stability of the Cauchy functional equation over $p$-adic fields. Real Anal. Exchange 31, 125-132 (2005/2006)

[10] Arkeryd, L., Cercignani, C.: On a functional equation arising in the kinetic theory of gases. Atti Accad. Naz. Lincei Cl. Sci. Fis. Mat. Natur. Rend. Lincei (9) Mat. Appl 1(2), 139-149 (1990)

[11] Badora, R., Chmieliński, J.: Decomposition of mappings approximately inner product preserving. Nonlinear Anal. 62, 1015-1023 (2005)

[12] Bajger, M.: On the composite Pexider equation modulo a subgroup. Publ. Math. Debrecen 64(1-2), 39-61 (2004)

[13] Baker, J.: The stability of the cosine equation. Proc. Am. Math. Soc. 80(3), 411416 (1980)

[14] Baker, J., Lawrence, J., Zorzitto, F.: The stability of the equation $f(x+y)=$ $f(x) f(y)$. Proc. Am. Math. Soc. 74(2), 242-246 (1979)

[15] Baron, K.: Orthogonality and additivity modulo a discrete subgroup. Aequationes Math. 70, 189-190 (2005)

[16] Baron, K.: On some orthogonally additive functions on inner product spaces. Annales Univ. Sci. Budapest. Sect. Comp. 40, 123-127 (2013)

[17] Baron, K.: On orthogonally additive functions with orthogonally additive second iterate. Comment. Math. 53(2), 401-404 (2013)

[18] Baron, K.: Orthogonally additive bijections are additive. Aequationes Math (2013). doi:10.1007/s00010-013-0242-6

[19] Baron, K., Forti, G.L.: Orthogonality and additivity modulo $\mathbb{Z}$. Results Math. 26, 205-210 (1994)

[20] Baron, K., Halter-Koch, F., Volkmann, P.: On orthogonally exponential functions. Arch. Math. (Basel) 64(5), 410-414 (1995)

[21] Baron, K., Kucia, A.: On regularity of functions connected with orthogonal additivity. Funct. Approx. Comment. Math. 26, 19-24 (1998)

[22] Baron, K., Rätz, J.: Orthogonality and additivity modulo a subgroup. Aequationes Math. 46(1-2), 11-18 (1993)

[23] Baron, K., Rätz, J.: On orthogonally additive mappings on inner product spaces. Bull. Polish Acad. Sci. Math. 43(3), 187-189 (1995)

[24] Baron, K., Volkmann, P.: On orthogonally additive functions. Publ. Math. Debrecen 52(3-4), 291-297 (1998)

[25] Beyer, W.A.: Approximately Lorentz Transformations and $p$-adic Fields. LA-DC9486, Los Alamos National Laboratory (1968)

[26] Birkhoff, G.: Orthogonality in linear metric spaces. Duke Math. J. 1, 169-172 (1935)

[27] Blanco, A., Turnšek, A.: On maps that preserve orthogonality in normed spaces. Proc. R. Soc. Edinb. Sect. A 136, 709-716 (2006)

[28] Blaschke, W.: Räumliche Variationsprobleme mit symmetrischer Transversalitätsbedingung. Ber. Verh. Sächs. Ges. Wiss. Leipzig. Math. Phys. Kl. 68, 5055 (1916)

[29] de Bruijn, N.G.: On almost additive functions. Colloq. Math. 15, 59-63 (1966)

[30] Brzdęk, J.: On functionals which are orthogonally additive modulo $\mathbb{Z}$. Results Math. 30, 25-38 (1996)

[31] Brzdęk, J.: On orthogonally exponential and orthogonally additive mappings. Proc. Am. Math. Soc. 125(7), 2127-2132 (1997) 
[32] Brzdęk, J.: On orthogonally exponential functionals. Pac. J. Math. 181(2), 247267 (1997)

[33] Brzdęk, J.: On measurable orthogonally exponential functions. Arch. Math. (Basel) 72(3), 185-191 (1999)

[34] Brzdęk, J.: On the isosceles orthogonally exponential mappings. Acta Math. Hungar. 87(1-2), 147-152 (2000)

[35] Brzdęk, J., Sikorska, J.: On a conditional exponential functional equation and its stability. Nonlinear Anal. 72, 2923-2934 (2010)

[36] Chacon, R.V., Friedman, N.: Additive functionals. Arch. Ration. Mech. Anal. 18, 230-240 (1965)

[37] Chmieliński, J.: Almost approximately inner product preserving mappings. Aequationes Math. 59, 214-221 (2000)

[38] Chmieliński, J.: On a singular case in the Hyers-Ulam-Rassias stability of the Wigner equation. J. Math. Anal. Appl. 289, 571-583 (2004)

[39] Chmieliński, J.: Linear mappings approximately preserving orthogonality. J. Math. Anal. Appl. 304, 158-169 (2005)

[40] Chmieliński, J.: On an $\varepsilon$-Birkhoff orthogonality. J. Inequal. Pure Appl. Math. 6(3), Art. 79 (2005)

[41] Chmieliński, J.: Stability of the orthogonality preserving property in finitedimensional inner product spaces. J. Math. Anal. Appl. 318, 433-443 (2006)

[42] Chmieliński, J.: Stability of the Wigner equation and related topics. Nonlinear Funct. Anal. Appl. 11, 859-879 (2006)

[43] Chmieliński, J.: Remarks on orthogonality preserving mappings in normed spaces and some stability problems. Banach J. Math. Anal. 1, 117-124 (2007)

[44] Chmieliński, J.: Orthogonality preserving property and its Ulam stability; Chapter 4. in: Functional Equations in Mathematical Analysis. Springer Optimization and Its Applications, vol. 52. Springer, New York, pp. 33-58 (2012)

[45] Chmieliński, J., Ger, R.: On mappings preserving inner product modulo an ideal. Arch. Math. (Basel) 73, 186-192 (1999)

[46] Chmieliński, J., Moslehian, M.S.: Approximately $C^{*}$-inner product preserving mappings. Bull. Korean Math. Soc. 45, 157-167 (2008)

[47] Chmieliński, J., Rätz, J.: Orthogonality equation almost everywhere. Publ. Math. Debrecen 52, 317-335 (1998)

[48] Chmieliński, J., Wójcik, P.: Isosceles-orthogonality preserving property and its stability. Nonlinear Anal. 72, 1445-1453 (2010)

[49] Chmieliński, J., Wójcik, P.: On a $\rho$-orthogonality. Aequationes Math. 80, 4555 (2010)

[50] Chmieliński, J., Wójcik, P.: $\rho$-orthogonality and its preservation-revisited. Recent Developments in Functional Equation and Inequalities, Banach Center Publications, vol. 99. Institute of Mathematic, Polish Academy of Sciences, Warszawa, pp. 17-30 (2013)

[51] Chudziak, J.: Approximate solutions of the Gołąb-Schinzel functional equation. J. Approx. Theory 136, 21-25 (2005)

[52] Davison, T.M.K., Ebanks, B.R.: Cocycles on cancellative semigroups. Publ. Math. Debrecen 46, 137-147 (1995)

[53] Day, M.M.: Some characterizations of inner-product spaces. Trans. Am. Math. Soc. 62, 320-337 (1947)

[54] Day, M.M.: Normed Linear Spaces. Springer, Berlin (1973)

[55] Dhombres, J.: Some Aspects of Functional Equations. Chulalongkorn University Press, Bangkok (1979)

[56] Diminnie, C.R.: A new orthogonality relation for normed linear spaces. Math. Nachr. 114, 197-203 (1983)

[57] Ding, G.G.: The approximation problem of almost isometric operators by isometric operators. Acta Math. Sci. (English Ed.) 8, 361-372 (1988) 
[58] Dragomir, S.S.: On approximation of continuous linear functionals in normed linear spaces. An. Univ. Timişoara Ser. Ştiinţ. Mat. 29, 51-58 (1991)

[59] Dragomir, S.S.: Continuous linear functionals and norm derivatives in real normed spaces. Univ. Beograd. Publ. Elektrotehn. Fak. Ser. Mat. 3, 5-12 (1992)

[60] Dragomir, S.S.: Semi-Inner Products and Applications. Nova Science Publishers Inc, Hauppauge (2004)

[61] Drewnowski, L., Orlicz, W.: On orthogonally additive functional. Bull. Acad. Polon. Sci. Sér. Sci. Math. Astronom. Phys. 16, 883-888 (1968)

[62] Drljević, H.: On the stability of the functional quadratic on $A$-orthogonal vectors. Publ. Inst. Math. (Beograd) (N.S.) 36(50), 111-118 (1984)

[63] Drljević, H.: On a functional which is quadratic on $A$-orthogonal vectors. Institut Mathématique. Publications. Nouvelle Série. (Beograd) (N. S), 54, 63-71 (1986)

[64] Drljević, H., Mavar, Z.: About the stability of a functional approximately additive on $A$-orthogonal vectors. Akad. Nauka Umjet. Bosne Hercegov. Rad. Odjelj. Prirod. Mat. Nauka 20, 155-172 (1982)

[65] Erdős, P.: On the distribution function of additive functions. Ann. Math. (2) 47, 120 (1946)

[66] Erdős, P.: Problem P310. Colloq. Math. 7, 311 (1960)

[67] Fechner, W.: On functions with the Cauchy difference bounded by a functional. III. Abh. Math. Sem. Univ. Hamburg 76, 57-62 (2006)

[68] Fechner, W., Sikorska, J.: Sandwich theorems for orthogonally additive functions. Inequal. Appl. Int. Ser. Numer. Math. 157, 269-281 (2008)

[69] Fechner, W., Sikorska, J.: On the stability of orthogonal additivity. Bull. Polish Acad. Sci. Math. 58, 23-30 (2010)

[70] Fochi, M.: Alcune equazioni funzionali condizionate sui vettori ortogonali. Rend. Sem. Mat. Univ. Politec. Torino 44, 397-406 (1986)

[71] Fochi, M.: Functional equations in A-orthogonal vectors. Aequations Math. 38, 2840 (1989)

[72] Fochi, M.: D'Alembert's functional equation on restricted domains. Aequationes Math. 52, 246-253 (1996)

[73] Fochi, M.: Characterization of special classes of solutions for some functional equations on orthogonal vectors. Aequationes Math. 59, 150-159 (2000)

[74] Fochi, M.: An alternative functional equation on restricted domain. Aequationes Math. 70, 201-212 (2005)

[75] Fochi, M.: An alternative-conditional functional equation and the orthogonally additive functionals. Aequationes Math. 78, 309-320 (2009)

[76] Fochi, M.: General solutions of two quadratic functional equations of Pexider type on orthogonal vectors. Abstr. Appl. Anal. (2012) Art. ID 675810

[77] Forti, G.L.: Hyers-Ulam stability of functional equations in several variables. Aequationes Math. 50, 143-190 (1995)

[78] Friedman, N., Katz, M.: Additive functional in $L^{p}$ spaces. Can. J. Math. 18, 12641271 (1966)

[79] Friedman, N., Katz, M.: A representation theorem for additive functionals. Arch. Ration. Mech. Anal. 21, 49-57 (1966)

[80] Găvruţa, P., Găvruţa, L.: Hyers-Ulam-Rassias stability of orthogonal additive mappings. Nonlinear analysis, 291-303, Springer Optim. Appl., vol. 68. Springer, New York (2012)

[81] Gel'fand, I.M., Vilenkin, N.: Generalized Functions. Some Applications of Harmonic Analysis, vol. 4. Fizmatgiz, Moscow (1961) [English transl., Academic Press, New York (1964)]

[82] Ger, R.: $n$-convex functions in linear spaces. Aequationes Math. 10, 172-176 (1974)

[83] Ger, R.: Almost additive functions on semigroups and a functional equation. Publ. Math. Debrecen 26, 219-228 (1979) 
[84] Ger, R.: Conditional Cauchy equation stemming from ideal gas theory. In: Proceedings of the Nineteenth International Symposium on Functional Equations, University of Waterloo, p. 22 (1981)

[85] Ger, R.: Superstability is not natural. Rocznik Nauk. Dydakt. Prace Mat. 13, 109123 (1993)

[86] Ger, R.: Remark 22. In: The Thirty-third International Symposium on Functional Equations, May 21-May 27, 1995, Caldes de Malavella. Aequationes Math., vol. 51, p. 177 (1996)

[87] Ger, R.: Orthogonalities in linear spaces and difference operators. Aequationes Math. 60, 268-282 (2000)

[88] Ger, R., Šemrl, P.: The stability of the exponential equation. Proc. Am. Math. Soc. 124, 779-787 (1996)

[89] Ger, R., Sikorska, J.: Stability of the orthogonal additivity. Bull. Polish Acad. Sci. Math. 43, 143-151 (1995)

[90] Ger, R., Sikorska, J.: On the Cauchy equation on spheres. Ann. Math. Sil. 11, 8999 (1997)

[91] Giles, J.R.: Classes of semi-inner-product spaces. Trans. Am. Math. Soc. 129, 436446 (1967)

[92] Godini, G.: Set-valued Cauchy functional equation. Rev. Roumaine Math. Pures Appl. 20, 1113-1121 (1975)

[93] Gouvêa, F.Q.: p-adic Numbers. Springer, Berlin (1997)

[94] Gudder, S., Strawther, D.: Orthogonality and nonlinear functionals. Bull. Am. Math. Soc. 80, 946-950 (1974)

[95] Gudder, S., Strawther, D.: Orthogonally additive and orthogonally increasing functions on vector spaces. Pac. J. Math. 58, 427-436 (1975)

[96] Gudder, S., Strawther, D.: A converse of Pythagoras' theorem. Am. Math. Monthly 84, 551-553 (1977)

[97] Heijnen, B., Goovaerts, M.J.: Additivity and premium calculation principles. Blätter der Deutschen Gesellschaft für Versich. Math. 17, 217-223 (1986)

[98] Hyers, D.H.: On the stability of the linear functional equation. Proc. Natl. Acad. Sci. USA 27, 222-224 (1941)

[99] Hyers, D.H., Isac, G., Rassias, Th.M.: Stability of Functional Equations in Several Variables. Birkhäuser, Basel (1998)

[100] Ilišević, D., Turnšek, A.: Approximately orthogonality preserving mappings on $C^{*}$ modules. J. Math. Anal. Appl. 341, 298-308 (2008)

[101] Ionică, I.: On linear operators preserving orthogonality. Ann. Ştiinţ. Univ. Al. I. Cuza Iaşi. Mat. (N.S.) 58, 325-332 (2012)

[102] James, R.C.: Orthogonality in normed linear spaces. Duke Math. J. 12, 291$302(1945)$

[103] James, R.C.: Inner products in normed linear spaces. Bull. Am. Math. Soc. 53, 559566 (1947)

[104] James, R.C.: Orthogonality and linear functionals in normed linear spaces. Trans. Am. Math. Soc. 61, 265-292 (1947)

[105] Jung, S.-M.: Hyers-Ulam-Rassias Stability of Functional Equations in Mathematical Analysis. Hadronic Press, Palm Harbor (2001)

[106] Jurkat, W.B.: On Cauchy's functional equation. Proc. Am. Math. Soc. 16, 683686 (1965)

[107] Kalton, N.J., Roberts, J.W.: Uniformly exhaustive submeasures and nearly additive set functions. Trans. Am. Math. Soc. 278, 803-816 (1983)

[108] Kátai, I.: A remark on additive arithmetical functions. Ann. Univ. Sci. Budapest. Eötvös Sect. Math. 10, 81-83 (1967)

[109] Kochanek, T.: Stability aspects of arithmetic functions. Acta Arith. 132, 87-98 (2008)

[110] Kochanek, T.: Stability aspects of arithmetic functions. II. Acta Arith. 139, 131146 (2009) 
[111] Kochanek, T.: Corrigendum to "Stability aspects of arithmetic functions, II" (Acta Arith. 139 (2009), 131-146). Acta Arith. 149, 83-98 (2011)

[112] Kochanek, T.: Stability of vector measures and twisted sums of Banach spaces. J. Funct. Anal. 264, 2416-2456 (2013)

[113] Kochanek, T., Lewicki, M.: Stability problem for number-theoretically multiplicative functions. Proc. Am. Math. Soc. 135, 2591-2597 (2007)

[114] Kochanek, T., Wyrobek, W.: Measurable orthogonally additive functions modulo a discrete subgroup. Acta Math. Hungar. 123(3), 239-248 (2009)

[115] Kochanek, T., Wyrobek-Kochanek, W.: Almost orthogonally additive functions. J. Math. Anal. Appl. 400, 1-14 (2013)

[116] Koehler, D., Rosenthal, P.: On isometries of normed linear spaces. Studia Math. 36, 213-216 (1970)

[117] Koldobsky, A.: Operators preserving orthogonality are isometries. Proc. R. Soc. Edinb. Sect. A 123, 835-837 (1993)

[118] Kolwicz, P., Płuciennik, R.: P-convexity of Orlicz-Bochner spaces. Proc. Am. Math. Soc. 126, 2315-2322 (1998)

[119] Kong, L., Cao, H.-X.: Stability of orthogonality preserving mapping and the orthogonality equation. J. Shaanxi Normal Univ. (Natural Science Edition) 36(5), 1014 (2008)

[120] Kuczma, M.E.: Problem 10381. Amer. Math. Monthly 101, 363 (1994). Solution 10381. Amer. Math. Monthly 104, 365-366 (1997)

[121] Lance, E.C.: Hilbert $C^{*}$-Modules. London Math. Soc. Lecture Note Series, vol. 210. Cambridge University Press, Cambridge (1995)

[122] Lawrence, J.: Orthogonality and additive mappings on normed linear spaces. Colloq. Math. 49, 253-255 (1985)

[123] Leaderman, H., McCrackin, F., Nakada, O.: Large longitudinal retarded elastic deformation of rubber like network polymers. II. Applications of a general formulation of nonlinear responses. Trans. Soc. Rheology 7, 111-123 (1963)

[124] Lumer, G.: Semi-inner-product spaces. Trans. Am. Math. Soc. 100, $29-43$ (1961)

[125] Maksa, Gy., Szabó, Gy., Székelyhidi, L.: Equations arising from the theory of orthogonally additive and quadratic functions. C. R. Math. Rep. Acad. Sci. Canada 10(6), 295-300 (1988)

[126] Martin, A., Mizel, V.: A representation theorem for non-linear functionals. Arch. Ration. Mech. Anal. 15, 353-367 (1964)

[127] Máté, A.: A new proof of a theorem of P. Erdős. Proc. Am. Math. Soc. 18, 159162 (1967)

[128] Matkowski, J.: Quadratic inequalities and a characterization of inner product. Math. Inequal. Appl. 15, 571-580 (2012)

[129] Miličić, P.M.: Sur la g-orthogonalité dans les espaces normés. Mat. Vesnik 39, 325334 (1987)

[130] Mirzavaziri, M., Moslehian, M.S.: A fixed point approach to stability of a quadratic equation. Bull. Braz. Math. Soc. (N.S.) 37, 361-376 (2006)

[131] Mizel, V.: Representation of nonlinear transformations on $L^{p}$ spaces. Bull. Am. Math. Soc. 75, 164-168 (1969)

[132] Mizel, V., Sundaresan, K.: Representation of additive and biadditive functionals. Arch. Ration. Mech. Anal. 30, 102-126 (1968)

[133] Mlak, W.: Hilbert Spaces and Operator Theory. Kluwer Academic Publisher Group, Dodrecht (1991)

[134] Mojškerc, B., Turnšek, A.: Mappings approximately preserving orthogonality in normed spaces. Nonlinear Anal. 73, 3821-3831 (2010)

[135] Moslehian, M.S.: On the orthogonal stability of the Pexiderized quadratic equation. J. Differ. Equ. Appl. 11, 999-1004 (2005)

[136] Moslehian, M.S., Rassias, Th.M.: Stability of functional equations in nonArchimedean spaces. Appl. Anal. Discrete Math. 1(2), 325-334 (2007) 
[137] Moszner, Z.: On the stability of functional equations. Aequationes Math. 77, 3388 (2009)

[138] Najati, A., Cho, Y.J.: Generalized Hyers-Ulam stability of the pexiderized Cauchy functional equation in non-Archimedean spaces. Fixed Point Theory Appl. (2011), Article ID 309026

[139] Paganoni, L., Rätz, J.: Conditional functional equations and orthogonal additivity. Aequationes Math. 50, 135-142 (1995)

[140] Park, C., Rassias, Th.M.: An additive functional equation in orthogonality spaces. In: Essays in Mathematics and Its Applications, pp. 335-367. Springer, Heidelberg (2012)

[141] Partington, J.R.: Orthogonality in normed spaces. Bull. Austral. Math. Soc. 33, 449455 (1986)

[142] Pawlik, B.: Approximately additive set functions. Colloq. Math. 54(1), 163164 (1987)

[143] Pinsker, A.: Sur une fonctionnelle dans l'espace de Hilbert. C. R. Acad. Sci. URSS N.S. 20, 411-414 (1938)

[144] Pólya, G., Szegő, G.: Problems and Theorems in Analysis. I. Springer, New York (1972)

[145] Protasov, V.Yu.: On stability of isometries in Banach spaces, Chapter 22. In: Functional Equations in Mathematical Analysis. Springer Optimization and Its Applications, vol. 52, pp. 273-285. Springer, Berlin (2012)

[146] Rassias, Th.M.: On the stability of the linear mapping in Banach spaces. Proc. Am. Math. Soc. 72, 297-300 (1978)

[147] Rätz, J.: On orthogonally additive mappings. In: Proceedings of the Eighteenth International Symposium on Functional Equations, University of Waterloo, pp. 22-23 (1980)

[148] Rätz, J.: On the conditional Cauchy functional equation of orthogonal additivity. Rend. Sem. Mat. Fis. Milano 53, 227-228 (1983)

[149] Rätz, J.: On orthogonally additive mappings. Aequationes Math. 28, 35-49 (1985)

[150] Rätz, J.: On orthogonally additive mappings. II. Publ. Math. Debrecen 35, 241249 (1988)

[151] Rätz, J.: On orthogonally additive mappings. III. Abh. Math. Sem. Univ. Hamburg 59, 23-33 (1989)

[152] Rätz, J.: Orthogonally additive mappings on free inner product $Z$-modules. Publ. Math. Debrecen 51, 97-110 (1997)

[153] Rätz, J.: Characterization of inner product spaces by means of orthogonally additive mappings. Aequationes Math. 58, 111-117 (1999)

[154] Rätz, J.: On a problem of J. Sikorska. In: Report of Meeting. The Thirty-seventh International Symposium on Functional Equations, May 16-23, 1999, Huntington. Aequationes Math., vol. 60, p. 184 (2000)

[155] Rätz, J.: A remark on a conditional cocycle equation. Dedicated to Professor Zenon Moszner on the occasion of his seventieth birthday. Rocznik Nauk. -Dydakt. Prace Mat. 17, 233-238 (2000)

[156] Rätz, J.: Cauchy functional equation problems concerning orthogonality. Aequationes Math. 62, 1-10 (2001)

[157] Rätz, J., Szabó, Gy.: On orthogonally additive mappings, IV. Aequationes Math. 38, 73-85 (1989)

[158] Rényi, A.: On a theorem of P. Erdős and its application in information theory. Mathematica (Cluj) 1, 341-344 (1959)

[159] Rivlin, R.: Stress relaxation in elastic materials for varying deformation. Armstrong Cork Co., Technical Report (1954)

[160] Robert, A.M.: A Course in p-adic Analysis. Springer, New York (2000)

[161] Roberts, B.D.: On the geometry of abstract vector spaces. Tôhoku Math. J. 39, 4259 (1934) 
[162] Schoenberg, I.J.: On two theorems of P. Erdös and A. Rényi. Ill. J. Math. 6, 53$58(1962)$

[163] Schwaiger, J.: Functional equations for homogeneous polynomials arising from multilinear mappings and their stability. Polish-Austrian Seminar on Functional Equations and Iteration Theory (Cieszyn, 1994). Ann. Math. Sil. 8, 157-171 (1994)

[164] Sikorska, J.: Stability of the orthogonal additivity. Doctorial dissertation (in Polish), Katowice (1997)

[165] Sikorska, J.: Problem 9. In: The Thirty-sixth International Symposium on Functional Equations, May 24-30, 1998, Brno, Aequationes Math., vol. 56, p. 304 (1998)

[166] Sikorska, J.: Orthogonal stability of the Cauchy equation on balls. Demonstratio Math. 33, 527-546 (2000)

[167] Sikorska, J.: Orthogonal stability of the Cauchy functional equation on balls in normed linear spaces. Demonstratio Math. 37, 579-596 (2004)

[168] Sikorska, J.: Generalized orthogonal stability of some functional equations. J. Inequal. Appl. (2006), Art. ID 12404

[169] Sikorska, J.: Generalized stability of the Cauchy and Jensen functional equations on spheres. J. Math. Anal. Appl. 345, 650-660 (2008)

[170] Sikorska, J.: On two conditional Pexider functional equations and their stabilities. Nonlinear Anal. 70, 2673-2684 (2009)

[171] Sikorska, J.: On a pexiderized conditional exponential functional equation. Acta Math. Hungar. 125(3), 287-299 (2009)

[172] Sikorska, J.: Exponential functional equation on spheres. Appl. Math. Lett. 23(2), 156-160 (2010)

[173] Sikorska, J.: On a direct method for proving the Hyers-Ulam stability of functional equations. J. Math. Anal. Appl. 372, 99-109 (2010)

[174] Sundaresan, K.: Additive functionals on Orlicz spaces. Studia Math. 32, 269$276(1969)$

[175] Sundaresan, K.: Orthogonality and nonlinear functionals on Banach spaces. Proc. Am. Math. Soc. 34, 187-190 (1972)

[176] Szabó, Gy.: On mappings orthogonally additive in the Birkhoff-James sense. Aequationes Math. 30, 93-105 (1986)

[177] Szabó, Gy.: On orthogonality spaces admitting nontrivial even orthogonally additive mappings. Acta Math. Hungar. 56, 177-187 (1990)

[178] Szabó, Gy.: Sesquilinear-orthogonally quadratic mappings. Aequationes Math. 40, 190-200 (1990)

[179] Szabó, Gy.: Continuous orthogonality spaces. Publ. Math. Debrecen 38, 311$322(1991)$

[180] Szabó, Gy.: A conditional Cauchy-equation on normed spaces. Publ. Math. Debrecen 42, 256-271 (1993)

[181] Szabó, Gy.: Isosceles orthogonally additive mappings and inner product spaces. Publ. Math. Debrecen 46, 373-384 (1995)

[182] Szabó, Gy.: Abstact of the talk. In: The thirty-third International Symposium on Functional Equations, May 21-May 27, 1995, Caldes de Malavella, Aequationes Math., vol 51, p. 159 (1996)

[183] Szabó, Gy.: Pythagorean orthogonality and additive mappings. Aequationes Math. 53, 108-126 (1997)

[184] Szabó, Gy.: Abstact of the talk. In: Report of Meeting. 5th International Conference on Functional Equations and Inequalities, Muszyna-Złockie, September 4-9, 1995. Rocznik Naukowo-Dydaktyczny WSP w Krakowie. Prace Matematyczne XV, p. 190 (1998)

[185] Szostok, T.: Modified version of Jensen equation and orthogonal additivity. Publ. Math. Debrecen 58, 491-504 (2001)

[186] Szostok, T.: On a generalized orthogonal additivity. Bull. Polish Acad. Sci. Math. 49, 395-408 (2001) 
[187] Szostok, T.: On some conditional functional equations. Ann. Math. Sil. 16, 6577 (2002)

[188] Truesdell, C., Muncaster, R.G.: Fundamentals of Maxwell's Kinetic Theory of a Simple Monatomic Gas. Academic Press, New York (1980)

[189] Turnšek, A.: On mappings approximately preserving orthogonality. J. Math. Anal. Appl. 336, 625-631 (2007)

[190] Turnšek, A.: A remark on orthogonally additive bijections. Aequationes Math. (2014). doi:10.1007/s00010-014-0261-y

[191] Ulam, S.M.: A Collection of Mathematical Problems. Interscience Publishers, New York (1960)

[192] Ulam, S.M.: Problems in Modern Mathematics. Science Editions. Wiley, New York (1964)

[193] Vajzović, F.: Über das Funktional $H$ mit der Eigenschaft: $(x, y)=0 \Rightarrow H(x+y)+$ $H(x-y)=2 H(x)+2 H(y)$. Glasnik Math. Ser. III 2, 73-81 (1967)

[194] Vladimirov, V.S., Volovich, I.V., Zelenov, E.I.: p-adic Analysis and Mathematical Physics. World Scientific Publishing Co., Singapore (1994)

[195] Wójcik, P.: Linear mappings preserving $\rho$-orthogonality. J. Math. Anal. Appl. 386, 171-176 (2012)

[196] Wójcik, P.: Operators preserving and approximately preserving orthogonality and similar relations. Doctorial dissertation (in Polish), Katowice (2013)

[197] Wójcik, P.: On certain basis connected with operator and its applications (submitted)

[198] Wyrobek, W.: Orthogonally additive functions modulo a discrete subgroup. Aequationes Math. 78, 63-69 (2009)

[199] Wyrobek, W.: Orthogonally Pexider functions modulo a discrete subgroup. Ann. Math. Sil. 26, 93-100 (2012)

[200] Yang, D.: Orthogonality spaces and orthogonally additive mappings. Acta Math. Hungar. 113, 269-280 (2006)

[201] Zamani, A., Moslehian, M.S.: Approximate Roberts orthogonality. Aequationes Math. (2013). doi:10.1007/s00010-013-0233-7

Justyna Sikorska

Institute of Mathematics

University of Silesia

Bankowa 14

40-007 Katowice, Poland

e-mail: justyna.sikorska@us.edu.pl

Received: March 29, 2014

Revised: June 16, 2014 